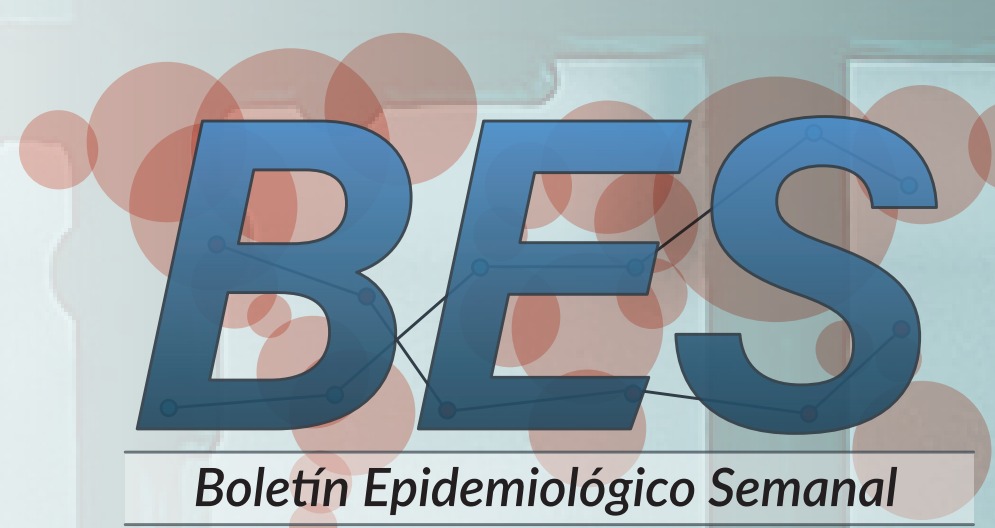

Semana epidemiológica 18

2 al 8 de mayo de 2021

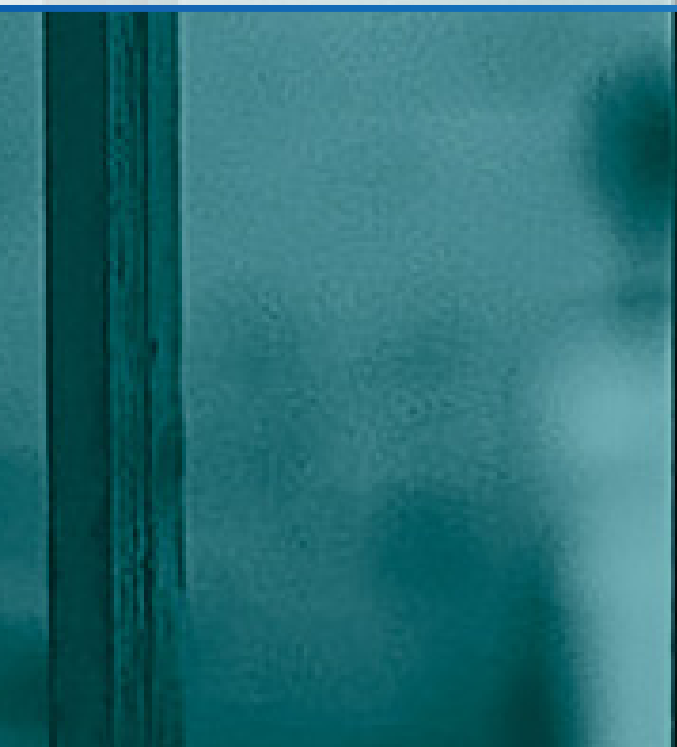

\section{COVID-19 en población}

privada de la libertad

\section{en los Establecimientos de}

Reclusión del Orden Nacional

de la libertad en los Establecimientos

de Reclusión del Orden Nacional (ERON)

Situación nacional tos en salud pública con mayor frecuencia y el cumplimiento de la tificación de las entidades territoriales.

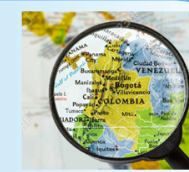
por eventos de interés en salud pública

Eventos trazadores Vigilancia en el comportamiento rutinario, siendo un indicador de brotes o emergencias en salud pública.

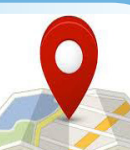

Brotes Actualidad a nivel internacional y nacional

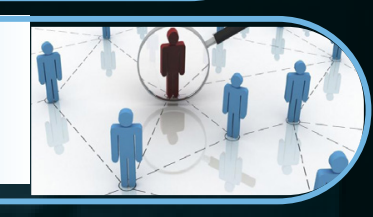

Situación COVID-19

Colombia

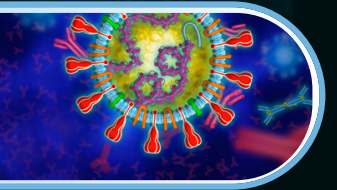

(ERON)

Tablas de mando por departamento Resume la vigilancia rutinaria, permite Resume la vigilancia rutinaria, perm
identificar brotes en salud pública.

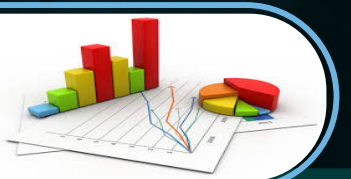


En Colombia, en el 2014 los establecimientos de reclusión a cargo del Instituto Nacional Penitenciario y Carcelario (INPEC) fueron caracterizados como Unidad Primaria Generadora de Datos (UPGD) ingresando así, como uno de los actores del Sistema Nacional de Vigilancia en Salud Pública (Sivigila). Desde el 2015 en trabajo conjunto entre el Instituto Nacional de Salud (INS) y el INPEC se realiza seguimiento a la notificación correspondiente a la Población Privada de la Libertad (PPL) a cargo del INPEC y desde el 2017 se articula el análisis con el Centro de Operaciones de Emergencias del INS, de acuerdo con los procesos del Sistema de Alerta Temprana (SAT) para la identificación del riesgo a través de la vigilancia basada en indicadores y en otras fuentes, para generar alertas en 5 eventos priorizados por su alta incidencia en esta población: tuberculosis, hepatitis B, intentos de suicidio, parotiditis y varicela individual.

No obstante, se mantiene la vigilancia rutinaria sobre todos los eventos de interés en salud pública (EISP) que ocurren al interior de los establecimientos de reclusión, incluyendo COVID-19 desde el 2020. Actualmente existen dos formas de identificar a la PPL en Sivigila, una de ellas a través de los códigos de caracterización asignados a cada centro penitenciario y la otra mediante el grupo poblacional carcelario de la ficha de notificación.

Según los datos del Sistema de Información del Sistema Penitenciario y Carcelario (SISIPEC WEB), con corte a 6 de mayo del 2021, se reporta una población de 97431 personas privadas de la libertad distribuidas en 132 establecimientos de reclusión a cargo del INPEC, ubicados en 28 departamentos de Colombia. El hacinamiento es de $19,5 \%$, el cual ha disminuido con respecto a mayo de 2020 que se encontraba en $39,5 \%$. El 28,1 \% de la población se encuentra entre los 18 y los 28 años, el 66,8 \% entre los 29 y los 60 años y el 5,1\% corresponde a mayores de 60 años. En los hombres se presenta el 90,5 \% (23 437) de los casos, mientras que en las mujeres el 9,5 \% (2 445) con una relación 13:1.

El primer caso de COVID-19 en PPL se reportó el 10 de abril de 2020 en el establecimiento penitenciario de la ciudad de Villavicencio-Me- ta. El total de casos confirmados con corte a 6 de mayo de 2021 a nivel nacional es de 25882 (tasa de incidencia: 26564 por 100000 habitantes) en PPL, de los cuales el 10,1\% (2 634) se presenta en personal de custodia y vigilancia (CCV) y auxiliares bachilleres. El número de personas privadas de la libertad recuperadas es 25675 (99,2\%), 147 han fallecido $(0,6 \%)$ y $60(0,2 \%)$ están activos. El mayor porcentaje de casos ocurrió en el grupo de 20 a 29 años con el 32,7 \% (8 464), seguido por el de 30 a 39 años con el $32.3 \%$ (8 371).

Las entidades territoriales con la mayor incidencia de COVID-19 en PPL por 100000 habitantes son Amazonas (79 120,88), Bolívar (70 312,5), Casanare (64 285,71), San Andrés (54 424,78), Boyacá (51 $654,8)$ y Risaralda $(49$ 133,29) (Figura 1.).

Figura 1. Tasas de incidencia de COVID-19 por entidad territorial en población privada de la libertad. Colombia, al 6 de mayo de 2021

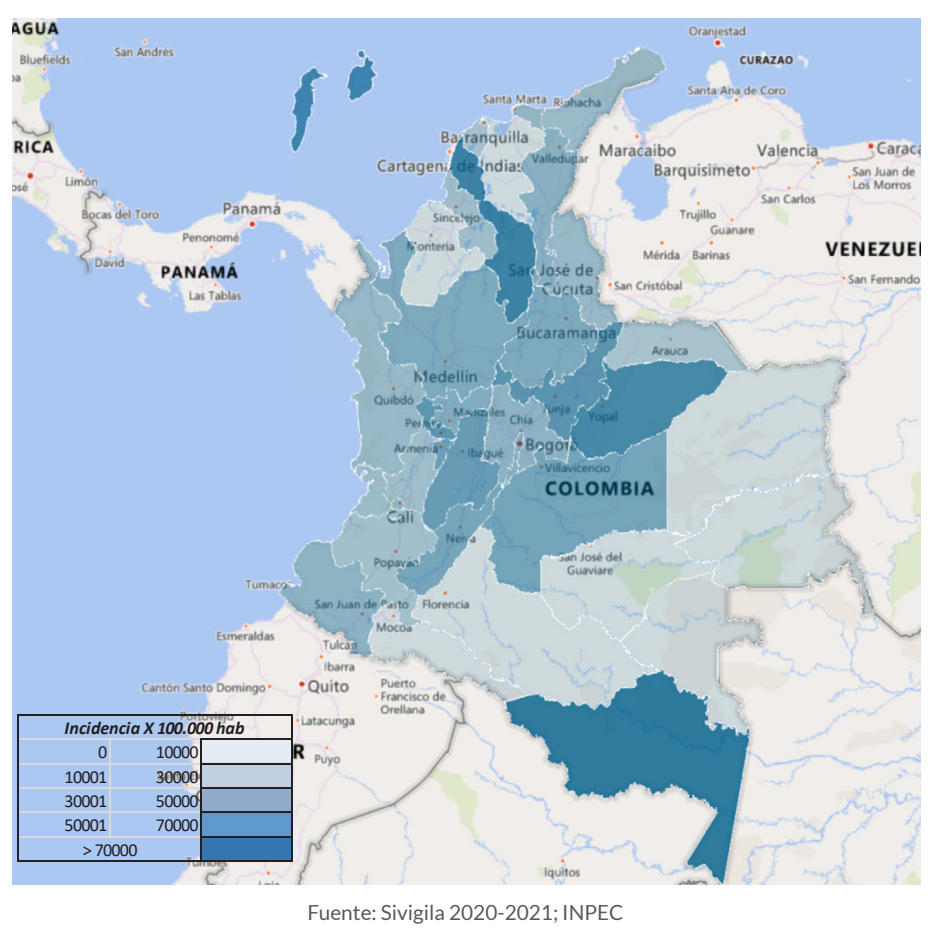

Tema central

Situación $\mathrm{Nal}$.

Mortalidad

Trazadores

Brotes

COVID-19

Tablas 
El porcentaje de positividad de COVID-19 en PPL es del 39,8\%, mientras que para los conglomerados en población general es de 37,2\%, lo que muestra una diferencia de $2,5 \%$. De las 31 entidades territoriales que cuentan con establecimientos de reclusión del orden nacional 15 (48,4 \%) superaron el porcentaje de positividad nacional. Las 5 entidades territoriales con el mayor porcentaje de positividad fueron en su orden: Amazonas con 75,8 \%, Bolívar 68,2 \%, Casanare $65,0 \%$, San Andrés y Providencia 58,6 \% y Risaralda 53,0 \%. Por establecimiento de reclusión, la positividad más alta ocurre en Guateque-Cundinamarca con el $96,9 \%$ seguido de El Guamo-Tolima con 91,9 \%, Riosucio-Caldas 91,1\%, Villeta-Cundinamarca con el 85,9\% y Granada-Meta con 85,2\%. El número de casos ha ido disminuyendo, así como la sobreocupación que se encontraba en 36916 PPL en abril de 2020, llegando a 15992 en mayo del 2021 (Figura 2.).

Figura 2. Comparación número de casos COVID-19 en población privada de la libertad vs. Sobrepoblación de los establecimientos de reclusión, Colombia, al 6 de mayo de 2021

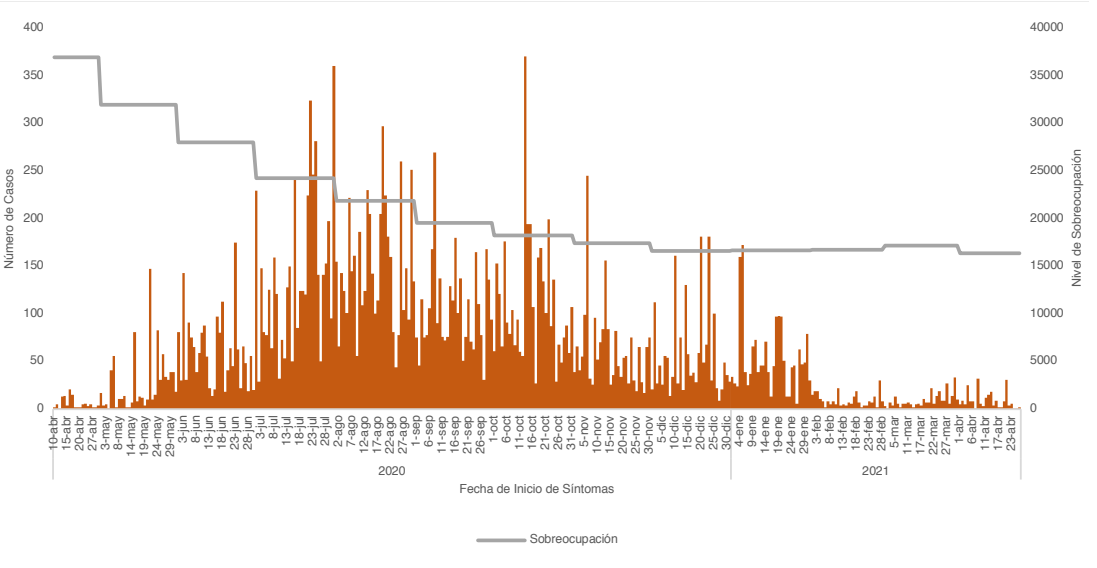

Fuente: Sivigila 2020-2021; INPEC

La letalidad de COVID-19 en la PPL a nivel nacional es de 0,6 \%, 10 entidades territoriales superaron este porcentaje en el siguiente orden: Barranquilla 2,2 \%, La Guajira 2,0 \%, Norte de Santander 1,3\%, Córdoba, Tolima y Valle del Cauca con 0,8\% cada uno, Caquetá, Nariño y Amazonas 0,7 \% cada uno y Bogotá 0,6 \% (Figura 3.)
Figura 3. Letalidad de COVID-19 por Establecimiento de Reclusión comparado con letalidad en población general y en privados de la libertad a nivel nacional, Colombia, al 6 de mayo de 2021

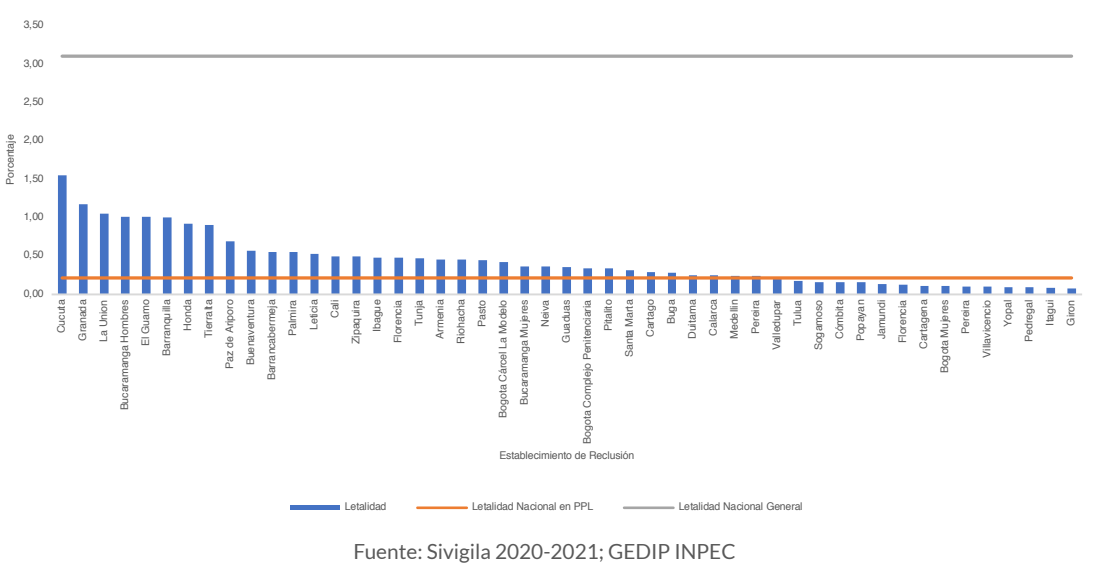

En el grupo de 80 años o más se registra la mayor letalidad (11,2\%) seguido del grupo de 70 a 79 años con 5,8\%. Sin embargo, el mayor número de fallecidos se encuentra en el grupo de 60 a 69 años (Figura 4.).

Figura 4. Fallecidos y tasa de letalidad de COVID-19 por grupo de edad población privada de la libertad, Colombia, al 6 de mayo de 2021

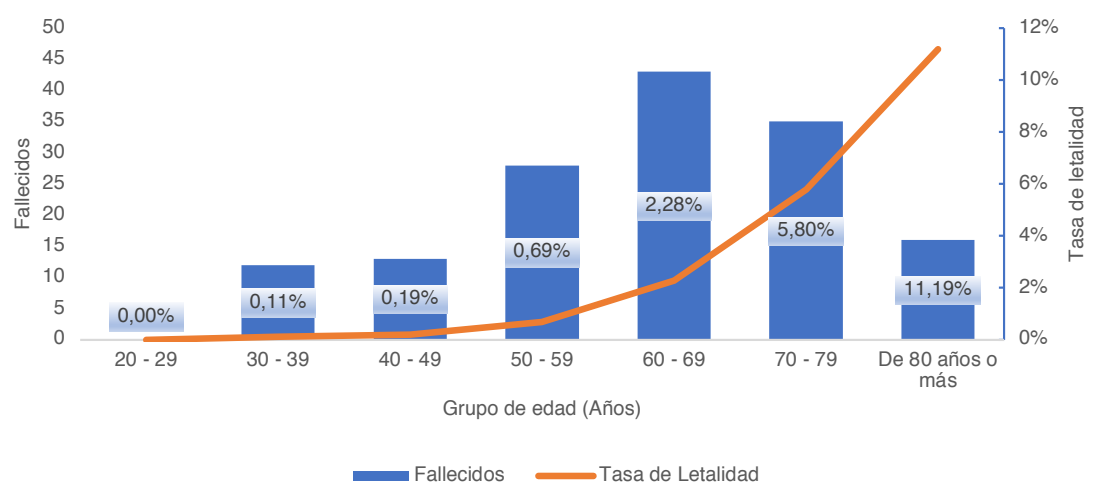

Fuente. Sivigila 2020-2021; INPEC

\section{Tema central \\ Situación Nal. \\ Mortalidad \\ Trazadores \\ Brotes}

COVID-19

Tablas 
La comorbilidad que se encontró con mayor frecuencia en la PPL que ha fallecido es hipertensión arterial (21\%), seguida de diabetes (13\%), VIH (6\%), diabetes e hipertensión (6\%), cáncer (4\%), enfermedad pulmonar obstructiva crónica-EPOC (4 \%), tuberculosis y EPOC-hipertensión ( $3 \%$ ) respectivamente y otras comorbilidades como enfermedad cardiovascular, enfermedad renal, asma, autoinmune con $2 \%$.

El tiempo promedio entre la fecha de inicio de síntomas y la consulta médica a nivel nacional es de 3,5 días; en PPL el $40 \%$ de las entidades territoriales superan este promedio, siendo Chocó, Cali y Boyacá las que registran los promedios más altos (10, 7 y 6 días respectivamente). El promedio de días entre la fecha de inicio de síntomas en la PPL y la fecha de diagnóstico fue de 11, mientras que en la población general el promedio reportado es de 9,5 días. En Colombia, el promedio de días entre la fecha de inicio de síntomas y la fecha de hospitalización es de 6,5 días, mientras que, en la PPL, el 31,0\% de las entidades territoriales superan el promedio nacional, siendo Caquetá la entidad que registra el mayor promedio con 14 días; así mismo, el promedio de días entre la fecha de inicio de síntomas y la fecha de defunción en el país es de 10,8; mientras que en la PPL el 82,1\% de las entidades territoriales superaron ese promedio, siendo Boyacá y Cali quienes registran el mayor promedio con 31 días cada uno.

Respecto a la vacunación contra COVID-19 con corte al 10 de mayo de 2021 en los establecimientos de reclusión del orden nacional se han aplicado 3247 primeras dosis en la población entre 60 y 80 años, distribuidas en 3210 para PPL, 19 personal del cuerpo de custodia, vigilancia y auxiliares bachilleres y 18 para funcionarios administrativos. La entidad territorial con mayor porcentaje de vacunación es Amazonas con el 87,8 \%, seguido por Antioquia con 5,6 \%, Tolima 3,9 $\%$, Cundinamarca 3,7 \% y Valle del Cauca con 3,4\%.
Entidades internacionales intervienen en los centros penitenciarios como parte de la misión humanitaria apoyando el desarrollo de acciones en salud pública; dentro de estas instituciones se destaca el Comité Internacional de la Cruz Roja (CICR) cuya misión en materia de detención es trabajar para que las condiciones y el trato hacia la PPL sea conforme a las normas internacionales y otros principios fundamentales que las protegen y realiza visitas a esta población con objetivo humanitario. Durante la pandemia de COVID-19, el CIRC ha continuado el apoyo al sistema penitenciario no solo a través del fortalecimiento de medidas de autocuidado en la PPL, la entrega de elementos de protección personal, también en el seguimiento al cumplimiento del decreto 546 de 2020 "Por medio del cual se adoptan medidas para sustituir la pena de prisión y la medida de aseguramiento de detención preventiva en establecimientos penitenciarios y carcelarios por la prisión domiciliaria y la detención domiciliaria transitorias en el lugar de residencia a personas que se encuentran en situación de mayor vulnerabilidad frente al COVID-19", además de la articulación continua con las autoridades sanitarias responsables de la salud en la PPL como el Ministerio de Salud y Protección Social (MSPS), INPEC y Unidad de Servicios Penitenciarios y Carcelarios (USPEC).
Tema central

Situación Nal.

Mortalidad

Trazadores

Brotes

COVID-19

Tablas 


\section{SITUACIÓN NACIONAL}

\section{Tablero de control del análisis de datos de la vigilancia}

Para el análisis de los eventos de mayor notificación en el país, se compara el valor observado en la semana epidemiológica correspondiente, con una línea de base de referencia, que está conformada con la información de esos eventos reportados en 15 intervalos de tiempo de al menos cinco años anteriores.

A partir de semana 14, se realizan las comparaciones con las vigencias 2015 a 2019, dada la atipicidad presentada para la vigencia 2020. Así se identifica que en la semana epidemiológica 18 los eventos infección respiratoria aguda grave (IRAG), morbilidad materna extrema (MME) y síflis gestacional, se encuentran por encima de lo esperado. Parotiditis, mortalidad perinatal y neonatal tardía, leishmaniasis cutánea, bajo peso al nacer, intoxicaciones y varicela individual, se encuentran por debajo de lo esperado. Los demás eventos están dentro del comportamiento histórico de la notificación (Figura 5).

Figura 5. Comparación de la notificación de casos de eventos priorizados, de alta frecuencia, según su comportamiento histórico. Colombia, a semana epidemiológica 18 de 2021

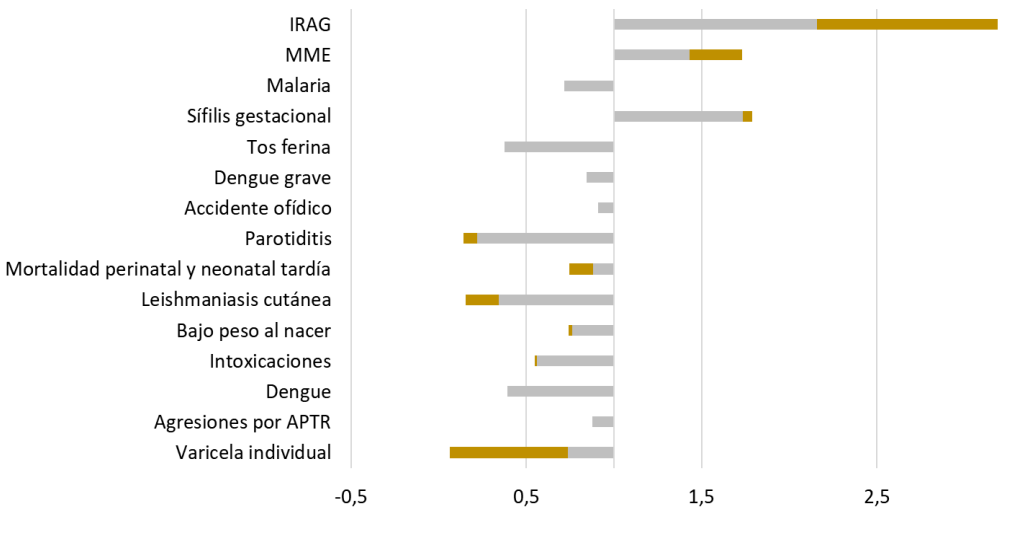

Fuente: Sivigila, Instituto Nacional de Salud, Colombia, 2021.
Para el análisis de los eventos de baja frecuencia o raros donde se asume que ocurren de manera aleatoria en el tiempo, se calcula la probabilidad de ocurrencia de cada evento según su comportamiento medio anterior, que para efectos de este análisis es el promedio de casos de 2015 a 2019. Con un nivel de confianza de $\mathrm{p}<0,05$ se determina si existen diferencias significativas entre lo observado y lo esperado.

A partir de semana 14, se realizan las comparaciones con las vigencias 2015 a 2019, dada la atipicidad presentada para la vigencia 2020. Para la semana epidemiológica 18, el evento adverso grave posterior a la vacunación, síflis congénita y mortalidad materna, presentan aumento significativo respecto a los valores esperados; los eventos sarampión, leptospirosis, rubeola, fiebre tifoidea y paratifoidea, lepra, tuberculosis farmacorresistente y mortalidad por dengue, presentan disminución significativa relacionada con el comportamiento de notificación histórico. Los demás eventos se encuentran dentro del comportamiento histórico (Tabla 1).

Tabla 1. Comparación de casos notificados de eventos priorizados, de baja frecuencia, según su comportamiento histórico, Colombia, a semana epidemiológica 18 de 2021

\begin{tabular}{lccc} 
Evento & Obser-vado & Esperado & Poisson \\
\hline EAPV & 28 & 14 & 0,00 \\
Síflis congénita & 29 & 22 & 0,03 \\
Mortalidad materna & 15 & 11 & 0,04 \\
& 1 & 17 & 0,00 \\
Sarampión & 10 & 59 & 0,00 \\
Leptospirosis & 29 & 61 & 0,00 \\
Rubeola & 4 & 12 & 0,00 \\
Lepra & 1 & 7 & 0,01 \\
Fiebre tifoidea y paratifoidea & 1 & 5 & 0,02 \\
Tuberculosis farmacorresistente & 3 & 8 & 0,03 \\
Mortalidad por dengue & 0 & 3 & 0,04 \\
Mortalidad por EDA 0-4 Años & 0 & 3 & 0,06 \\
Mortalidad por IRA & 10 & 13 & 0,08 \\
Leishmaniasis mucosa & 0 & 2 & 0,09 \\
\hline
\end{tabular}

Fuente: Sivigila, Instituto Nacional de Salud, Colombia, 2021
Tema central

Situación Nal.

Mortalidad

Trazadores

Brotes

COVID-19

Tablas instituto SALUD 


\section{Cumplimiento en la notificación}

Para esta semana la notificación recibida por el Instituto Nacional de Salud correspondiente a las unidades notificadoras departamentales y distritales (UND) fue del $100 \%$, permaneció igual comparándola con la semana anterior, así como con la misma semana del 2020.

El reporte de las unidades notificadoras municipales (UNM) a nivel nacional fue 99,9\% (1116 / 1117 UNM), en tanto se presentó silencio en el municipio La Pedrera del departamento de Amazonas. Se observa una disminución en comparación con lo presentado en la semana 17 y aumentó frente a la misma semana de 2020 (99,8\%). No obstante, el país cumplió con la meta del $97 \%$.

El cumplimiento de las Unidades Primarias Generadoras de Datos (UPGD) esta semana fue de 96,5 \% (5 033 / 5213 UPGD); disminuyó $1,1 \%$ frente a la semana anterior y aumentó en $2,1 \%$ con respecto a la semana 18 de 2020. A excepción de Nariño, todas las entidades territoriales cumplieron con la meta para este nivel de información. El país cumplió con la meta para la notificación de UPGD.

A continuación se muestra el comportamiento histórico de la notificación por semanas vigencias 2020 y 2021, los cuales se representan en puntos consecutivos y líneas sólidas, respectivamente; se observa las UPGD caracterizadas (líneas amarilla y lila), su comportamiento en la notificación (líneas azul y naranja) y su cumplimiento respecto la notificación de casos (meta $90 \%$ ) (líneas verde y gris); para ambas vigencias en semana 18 se evidencia el cumplimiento del número mínimo de UPGD que deben notificar (Figura 6).

Figura 6. Cumplimiento de la notificación por UPGD

Colombia, semanas epidemiológicas 1 a 18 de 2021
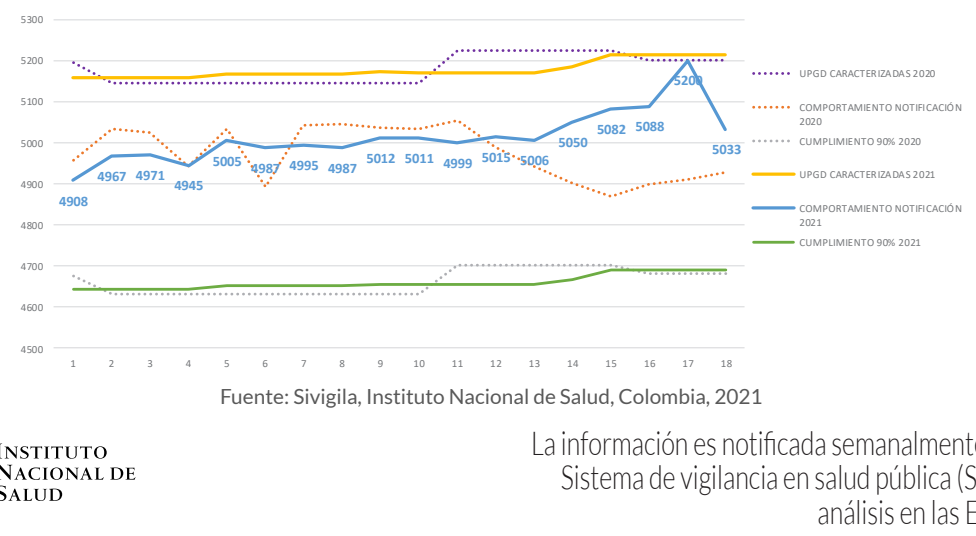

Fuente: Sivigia, instiktoNacionateSald, colonba, 2021
Tema central

Situación Nal.

Mortalidad

Trazadores

Brotes

COVID-19

Tablas 2020 se notificaron 5 casos. entidades territoriales de Córdoba, Magdalena, Santa Marta y Sucre en comparación con el histórico notificado a semana epidemiológica 18 entre 2018 a 2020, mientras que, en Vichada se observó una disminución. En las demás entidades no se observaron variaciones.

\section{Mortalidad por enfermedad diarreica aguda}

Para esta semana no se notificaron muertes probablemente asociadas a enfermedad diarreica aguda en menor de 5 años. Para la misma semana epidemiológica de 2020 se notificó 1 caso.

Para esta semana se observó un aumento en el número de casos en las entidades territoriales de Atlántico, Casanare, Santander y Sucre en comparación con el histórico notificado a semana epidemiológica 18 entre 2017 a 2020, mientras que, en Chocóy Antioquia se observóuna disminución. En las entidades territoriales restantes no se observaron variaciones. 
Para el análisis de los datos se tomó el comportamiento de cada uno de los eventos a semana epidemiológica 18 entre 2017 a 2020 (histórico) y se comparó con los casos observados a la misma semana epidemiológica del 2021. La razón esperada siempre será 1 y la significancia estadística estará dada por el valor de $\mathrm{p} \leq 0,05$ para identificar las entidades territoriales que presentan variaciones estadísticamente significativas.

\section{Mortalidad materna}

En la semana epidemiológica 18 de 2021 se notificaron 182 muertes maternas, 139 corresponden a mortalidad materna temprana (ocurridas durante el embarazo, parto y hasta los 42 días de terminada la gestación), 31 tardías (ocurridas desde el día 43 hasta un año de terminada la gestación) y 12 por causas coincidentes (lesiones de causa externa). Se observa un aumento del $31,1 \%$ en la mortalidad materna temprana respecto al 2020 (Tabla 2).

Tabla 2. Mortalidad materna según tipo de muerte, Colombia, semana epidemiológica 18, 2019 a 2021

\begin{tabular}{ccccc} 
& \multicolumn{3}{c}{ Tino de muerte } & Total \\
\cline { 2 - 4 } & Temprana & Tardía & Coincidente & 189 \\
2019 & 105 & 55 & 29 & 180 \\
2020 & 106 & 64 & 10 & 182 \\
2021 & 139 & 31 & 12 & 182 \\
\hline & Fuente: Sivigila, Instituto Nacional de Salud, Colombia, 2019-2021
\end{tabular}

La razón nacional preliminar de mortalidad materna a semana epidemiológica 18 es de 57,3 muertes por cada 100000 nacidos vivos. La razón de mortalidad materna superior a 100 muertes por cada 100000 nacidos vivos se observó en las entidades territoriales de Santa Marta, Magdalena, Chocó, Guaviare, Casanare y Risaralda (Tabla 3).
Tabla 3. Razón de mortalidad materna según entidad territorial de residencia, Colombia, semana epidemiológica 18 de 2021

\begin{tabular}{|c|c|c|}
\hline $\begin{array}{l}\text { Entidad territorial de } \\
\text { residencia }\end{array}$ & Número de casos & $\begin{array}{l}\text { Razón de MM por } 100000 \\
\text { nacidos vivos }\end{array}$ \\
\hline Santa Marta & 8 & 225,2 \\
\hline Magdalena & 10 & 187,8 \\
\hline Chocó & 4 & 184,7 \\
\hline Guaviare & 1 & 184,5 \\
\hline Casanare & 3 & 122,1 \\
\hline Risaralda & 4 & 105,3 \\
\hline La Guajira & 8 & 97,9 \\
\hline Bolívar & 5 & 83,6 \\
\hline Meta & 5 & 80,5 \\
\hline Nariño & 5 & 76,6 \\
\hline Barranquilla & 6 & 74,2 \\
\hline Cali & 7 & 72,2 \\
\hline Valle del Cauca & 5 & 66,5 \\
\hline Norte de Santander & 6 & 66,0 \\
\hline Córdoba & 6 & 63,5 \\
\hline Arauca & 1 & 59,8 \\
\hline Buenaventura & 1 & 57,7 \\
\hline $\begin{array}{l}\text { Colombia } \\
\text { Co }\end{array}$ & 139 & 57,3 \\
\hline Atlántico & 4 & 56,5 \\
\hline Putumayo & 1 & 54,7 \\
\hline Cesar & 4 & 46,3 \\
\hline Antioquia & 13 & 45,8 \\
\hline Huila & 3 & 43,8 \\
\hline Bogotá & 13 & 39,4 \\
\hline Sucre & 2 & 38,2 \\
\hline Boyacá & 2 & 37,1 \\
\hline Cundinamarca & 5 & 35,4 \\
\hline Cartagena & 2 & 32,3 \\
\hline $\begin{array}{l}\text { Tolima } \\
\text { Toll }\end{array}$ & 2 & 31,8 \\
\hline Caldas & 1 & 31,3 \\
\hline Santander & 2 & 18,9 \\
\hline
\end{tabular}

Tema centra

Situación Nal.

Mortalidad

Trazadores

Brotes

COVID-19

Tablas

Para la semana epidemiológica 18 de 2021 se observó un aumento significativo en las muertes maternas tempranas comparado con el promedio histórico en las entidades territoriales de Arauca, Bogotá, Guaviare, Magdalena, Santa Marta, Valle del Cauca y Cali y disminución en la entidad territorial de Cauca (Tabla 4).

Tabla 4. Entidades territoriales con comportamientos inusuales de mortalidad materna temprana respecto al promedio 2017-2020, Colombia, semana epidemiológica 18 de 2021

$\begin{array}{cccc}\text { Entidad territorial de residencia } & \text { Valor observado } & \text { Valor histórico } & \text { Poisson } \\ \text { Arauca } & 1 & 0 & 0,000 \\ \text { Bogotá } & 13 & 8 & 0,030 \\ \text { Cauca } & 0 & 3 & 0,050 \\ \text { Guaviare } & 1 & 0 & 0,000 \\ \text { Magdalena } & 10 & 5 & 0,018 \\ \text { Santa Marta } & 8 & 2 & 0,001 \\ \text { Valle del Cauca } & 5 & 1 & 0,003 \\ \text { Cali } & 7 & 3 & 0,022 \\ & \text { Fuente: Sivigila, Instituto Nacional de Salud, Colombia, 2017-2021 }\end{array}$


Encuanto a las causas de muerte materna temprana el $18,0 \%$ corresponde a causas directas y el $22,3 \%$ a causas indirectas. Las principales causas de muerte materna directa corresponden a trastorno hipertensivo asociado al embarazo con el 7,2\% y la hemorragia obstétrica con el 5,0\%; la principal causa de muerte indirecta es neumonía por Covid 19 con el 9,4\% (Tabla 5).

Tabla 5. Mortalidad materna temprana por tipo y causa principal agrupada, Colombia, semanas epidemiológicas 01 a 18 de 2021

$\begin{array}{lrr}\text { Causa agrupada } & \text { Casos } & \\ \text { DIRECTA } & \mathbf{2 5} & \mathbf{1 8 , 0} \\ \text { Trastorno hipertensivo asociado al embarazo } & 10 & 7,2 \\ \text { Hemorragia obstétrica } & 7 & 5,0 \\ \text { Sepsis relacionada con el embarazo } & 4 & 2,9 \\ \text { Evento tromboembolico como causa básica } & 3 & 2,2 \\ \text { Otras causas directas } & 1 & 0,7 \\ \text { INDIRECTA } & \mathbf{3 1} & \mathbf{2 2 , 3} \\ \text { Otras causas indirectas: Neumonía por COVID 19 } & 13 & 9,4 \\ \text { Otras causas indirectas } & 9 & 6,5 \\ \text { Sepsis no obstétrica: Neumonía } & 8 & 5,8 \\ \text { Sepsis no obstétrica } & 1 & 0,7 \\ \text { ENESTUDIO } & \mathbf{8 3} & \mathbf{5 9 , 7} \\ & & \end{array}$

Fuente: Sivigila, Instituto Nacional de Salud, Colombia, 2021

Para el análisis de los comportamientos inusuales, por ser la mortalidad materna un evento de baja frecuencia, se usa la distribución de probabilidades de Poisson por medio de la estimación de la probabilidad de ocurrencia del evento según su comportamiento medio entre el 2017 y 2020.

\section{Mortalidad perinatal y neonatal tardía}

A semana epidemiológica 18 de 2021, se han notificado 2789 casos de muerte perinatal y neonatal tardía (MPNT) de los cuales 36 corresponden a casos residentes en el exterior por lo que no se incluyen en el análisis. En esta semana se notificaron 172 casos, 130 corresponden a esta semana y 42 a notificaciones tardías.

En la semana epidemiológica analizada la razón preliminar nacional de mortalidad perinatal y neonatal tardía es de 11,1 muertes por cada 1000 nacidos vivos, en la tabla 1 se observa que en 23 entidades territoriales la razón es superior a la del país. Las 5 entidades territoriales con las razones más altas son Guainía $(30,6)$, Chocó $(21,7)$, Arauca (18,4), Córdoba $(17,7)$ y Barranquilla $(17,3)$.
Tabla 6. Número de casos y razón de mortalidad perinatal y neonatal tardía por entidad territorial de residencia, Colombia, semanas epidemiológicas 01 a 18 de 2021

\begin{tabular}{|c|c|c|}
\hline $\begin{array}{l}\text { Entidad territorial de } \\
\text { residencia }\end{array}$ & $\begin{array}{l}\text { Número de } \\
\text { casos }\end{array}$ & $\begin{array}{l}\text { Razón de Mortalidad perinatal y neonata } \\
\text { tardía por } 1000 \text { nacidos vivos }\end{array}$ \\
\hline Guainía & 12 & 30,6 \\
\hline Chocó & 48 & 21,7 \\
\hline Arauca & 31 & 18,4 \\
\hline Córdoba & 168 & 17,7 \\
\hline Barranquilla & 140 & 17,3 \\
\hline Vichada & 10 & 16,5 \\
\hline Bolívar & 97 & 16,1 \\
\hline Sucre & 82 & 15,6 \\
\hline Putumayo & 26 & 14,2 \\
\hline Buenaventura & 25 & 14,2 \\
\hline San Andrés y Providencia & 4 & 13,7 \\
\hline Casanare & 34 & 13,7 \\
\hline La Guajira & 112 & 13,7 \\
\hline Cauca & 93 & 13,1 \\
\hline Vaupés & 3 & 12,9 \\
\hline Risaralda & 49 & 12,8 \\
\hline Quindío & 26 & 12,2 \\
\hline Magdalena & 64 & 12,0 \\
\hline Cartagena & 74 & 11,9 \\
\hline Antioquia & 337 & 11,9 \\
\hline Atlántico & 83 & 11,7 \\
\hline Caquetá & 29 & 11,4 \\
\hline Cundinamarca & 159 & 11,2 \\
\hline Colombia & 2753 & 11,1 \\
\hline Guaviare & 6 & 11,0 \\
\hline Caldas & 35 & 10,9 \\
\hline Nariño & 71 & 10,8 \\
\hline Cesar & 90 & 10,4 \\
\hline Boyacá & 56 & 10,4 \\
\hline Tolima & 64 & 10,1 \\
\hline Santa Marta & 34 & 9,6 \\
\hline Amazonas & 4 & 9,4 \\
\hline Norte de Santander & 85 & 9,3 \\
\hline Huila & 62 & 9,0 \\
\hline Meta & 56 & 9,0 \\
\hline Cali & 82 & 8,5 \\
\hline Valle del Cauca & 63 & 8,4 \\
\hline Bogotá & 269 & 8,1 \\
\hline Santander & 70 & 6,6 \\
\hline
\end{tabular}

Tema central

Situación $\mathrm{Nal}$

Mortalidad

Trazadores

Brotes

COVID-19

Tablas 
Según el momento de ocurrencia de la muerte la mayor proporción son muertes perinatales anteparto con 47,8 \% (1 315), seguido de neonatales tempranas con $27,7 \%$ (762), neonatales tardías con 14,7 $\%$ (404) y perinatales intraparto con 9,8 \% (271).

De acuerdo con las causas de muerte agrupadas se observó la mayor proporción en: otras causas de muerte $26,6 \%$, principalmente trastornos de origen perinatal y placentarios, seguido por prematuridad-inmaturidad $24,2 \%$ y asfixia y causas relacionadas $20,4 \%$ (Figura 7 ).

Figura 7. Proporción de causas de muertes perinatales y neonatales tardías por causas de muerte agrupadas, Colombia, semanas epidemiológicas 01 a 18 de 2021

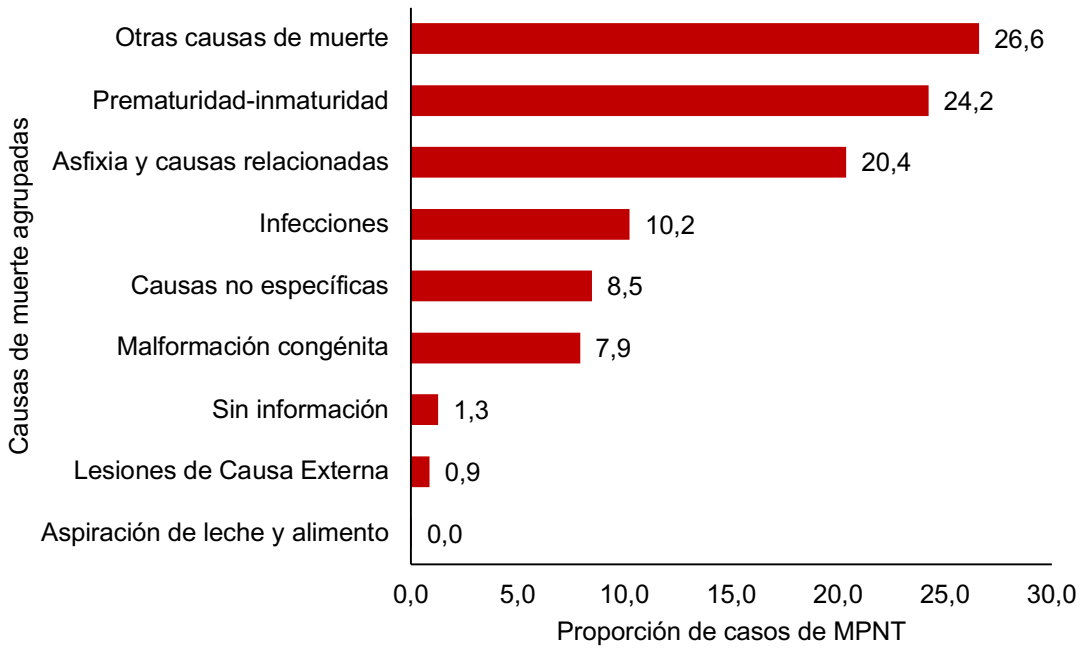

Fuente: Sivigila, Instituto Nacional de Salud, Colombia, 2021 (Datos preliminares).
En lo observado en la semana de análisis, en comparación con lo notificado entre 2016 y 2021, se presenta un decremento en la notificación en Cali, Cesar, Huila, Nariño y Valle del Cauca. Comportamiento que podría ser explicado por el subregistro en la notificación de las muertes a Sivigila, respecto al número de muertes certificadas en el Registro Único de Afiliados a la Protección Social-RUAF.

Dado que el evento tiene una alta frecuencia en notificación y se cuenta con una línea de base estable de más de cinco años, se realiza el análisis de los comportamientos inusuales a través del método: Morbidity and Mortality Weekly Report (MMWR) del Center for Disease Control and Prevention $(C D C)$. Este se basa en la comparación del comportamiento actual del evento en el último periodo epidemiológico (valor observado) con el resultado del promedio de 15 periodos históricos alrededor de ese periodo de evaluación, la ventana histórica de estimación contempla los cinco años previos al año de análisis (Coutin G, Borges J, Batista R, Feal P, Suárez B. Método para el análisis del comportamiento observado de enfermedades seleccionadas con relación al comportamiento histórico. Rev Cubana Hig Epidemiol. 2000;38(3):157-66).

\section{Tema centra}

Situación Nal.

Mortalidad

Trazadores

Brotes

COVID-19

Tablas 


\section{EVENTOS TRAZADORES}

\section{Infección respiratoria aguda}

A nivel nacional se identifica disminución en la notificación para la morbilidad por infección respiratoria aguda (IRA) en los servicios de consulta externa y urgencias; se presenta incremento en las hospitalizaciones por IRAG en sala general y en unidad de cuidados intensivos e intermedios (tabla 7).

Tabla 7. Notificación morbilidad por Infección Respiratoria Aguda por tipo de servicio en Colombia, semanas epidemiológicas 01 a 18, 2020 y 2021

\begin{tabular}{lrrrl} 
Tipo de servicio & 2020 a semana 18 & 2021 a semana 18 & \multicolumn{2}{c}{ Variación } \\
Consultas externas y urgencias & 2042571 & 1395620 & 31,7 & Disminución \\
Hospitalizaciones en sala general & 64938 & 85226 & 31,2 & Aumento \\
Hospitalizaciones en UCl & 7985 & 29092 & 264,3 & Aumento \\
\hline
\end{tabular}

A semana epidemiológica 18 de 2021, en Colombia se han notificado 1395620 consultas externas y urgencias por IRA, presentando disminución frente a lo notificado a la misma semana de los tres años anteriores con el 31,7 \% comparado con 2020, 35,2 \% frente a 2019 y $38,2 \%$ frente a 2018 . Teniendo en cuenta el comportamiento de notificación de los últimos siete años, se presenta disminución en 22 entidades territoriales; Córdoba, Guainía, La Guajira y Santa Marta presentan incremento; Antioquia, Arauca, Barranquilla, Bolívar, Cartagena, Casanare, Chocó, Cundinamarca, Magdalena, Risaralda, Sucre y Valle del Cauca no presentan comportamientos inusuales.

Por grupos de edad, los adultos de 20 a 39 años representan el 31,7 \% (441 909), seguido por el de 40 a 59 años con el 21,1 \% (294 496). La mayor proporción de consultas externas y de urgencias por IRA sobre el total de consultas por todas las causas se presenta en los niños de 1 año con el 8,5\% seguido de los menores de 1 año con el 8,2 \%.

En el canal endémico las consultas externas y urgencias por IRA en las semanas trascurridas del año se ubican por debajo del umbral estacional, a partir de la semana 02 por debajo del límite inferior hasta la semana
13; desde la semana 14 se observan en zona de seguridad, por encima del límite inferior (figura 8).

Figura 8. Canal endémico de consultas externas y urgencias por infección respiratoria aguda, Colombia, semanas epidemiológicas 01 a 18, entre 2014 y 2021

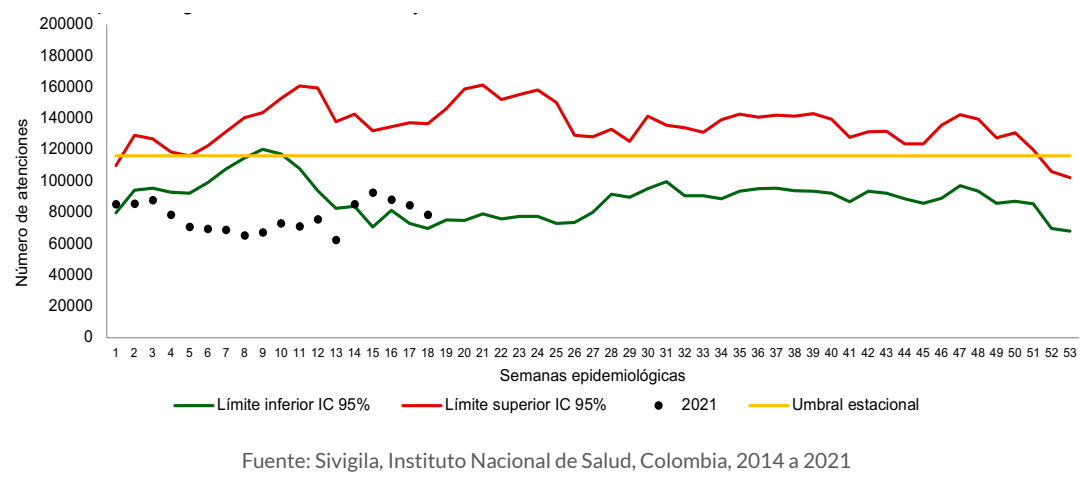

Senotificaron85226hospitalizacionespor IRAGensalageneral, presentando incremento frente a lo notificado a la misma semana de los tres años anteriores con el $31,2 \%$ frente a 2020,11,5\% comparado con 2019 y del 5,0 $\%$ frente a 2018. Teniendo en cuenta el comportamiento de notificación de los últimos siete años, se presenta disminución en 9 entidades territoriales, entre las cuales resaltan Vaupés, Caquetá, Quindío y Cali; e incremento en 14 entidades territoriales resaltando Barranquilla, Magdalena, Antioquia, Casanare y Cundinamarca; no presentan comportamientos inusuales 15 entidades territoriales.

Por grupos de edad, los adultos de más de 60 años representan el 37,9 \% (32 283), seguido por los de 40 a 59 años con el 22,1 \% (18 838). La mayor proporción de hospitalizaciones en sala general por IRAG sobre el total de hospitalizaciones por todas las causas se presenta en los niños de 1 año con el $17,1 \%$, seguido por los niños de 2 a 4 años con el $13 \%$.

En el canal endémico durante las cuatro primeras semanas del año las hospitalizaciones por IRAG en sala general se ubicaron por encima del límite superior histórico esperado y el umbral estacional, para la semana 05 se observa un descenso por debajo del umbral estacional, sin variación para las semanas 06 a 08; de las semanas 09 a 12 se 
ubican en zona de éxito, por debajo del límite interior, para las semanas 13 a 18 se observa un aumento ubicándose por encima del umbral estacional; en las últimas dos semanas se observa un incremento por encima del límite superior histórico establecido (figura 9).

Figura 9. Canal endémico de hospitalizaciones por infección respiratoria aguda grave en sala general, Colombia, semanas epidemiológicas 01 a 18, entre 2014 y 2021

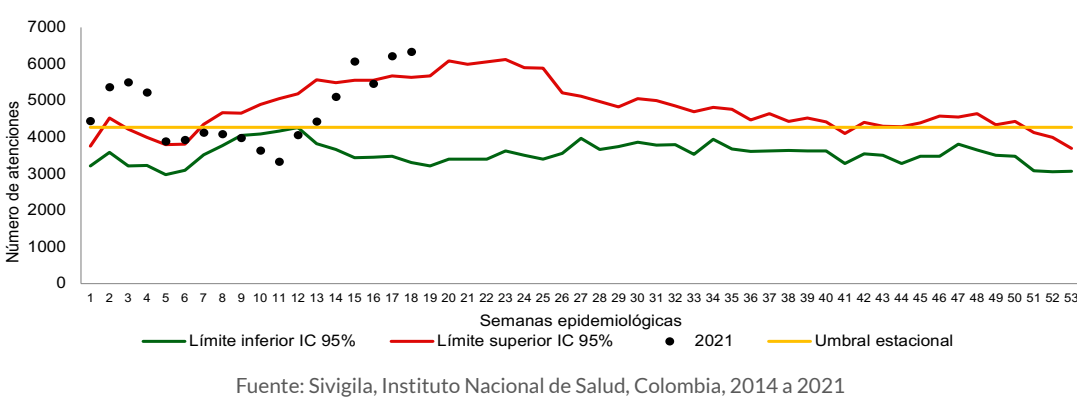

Se notificaron 29092 hospitalizaciones por IRAG en unidad de cuidados intensivos - $\mathrm{UCl}$ e intermedios, presentando incremento frente a lo notificado a la misma semana de los tres años anteriores con el $264,3 \%$ frente a $2020,319,6 \%$ comparado con 2019 y 318,7 \% frente a 2018. Se presenta incremento en 30 entidades territoriales, entre las cuales resaltan Valle del Cauca, Bolívar, Caquetá, Antioquia, Cesar y Nariño; Arauca presenta disminución y no presentan comportamientos inusuales: Amazonas, Cali, Chocó, Guainía, Guaviare, Vaupés y Vichada.

Por grupos de edad, los adultos de más de 60 años representan el 54,1 \% (15 725), seguido por los de 40 a 59 años con el $27,5 \%$ (8 007) y los de 20 a 39 años con el 8,3\% (2428). La mayor proporción de hospitalizaciones por IRAG en $\mathrm{UCl}$ e intermedios sobre el total de hospitalizaciones en $\mathrm{UCl}$ por todas las causas se presenta en el grupo de mayores de 60 años con el $30,3 \%$, seguido por el de 40 a 59 años con el 30,8\%.

Para las 5 primeras semanas las hospitalizaciones por IRAG en $\mathrm{UCI}$ e intermedios superaron el promedio histórico y el límite superior de las semanas 01 a 53 de 2020, adicionalmente se presenta un incremento progresivo hasta la tercera semana y un descenso por siete semanas; en las últimas ocho semanas se observa incremento por encima del umbral estacional y a partir de la semana 14 se supera nuevamente el límite superior (figura 10).

Figura 10. Comportamiento de hospitalizaciones por infección respiratoria aguda grave en unidades de cuidados intensivos, Colombia, semanas epidemiológicas 01 a 18, entre 2020 y 202

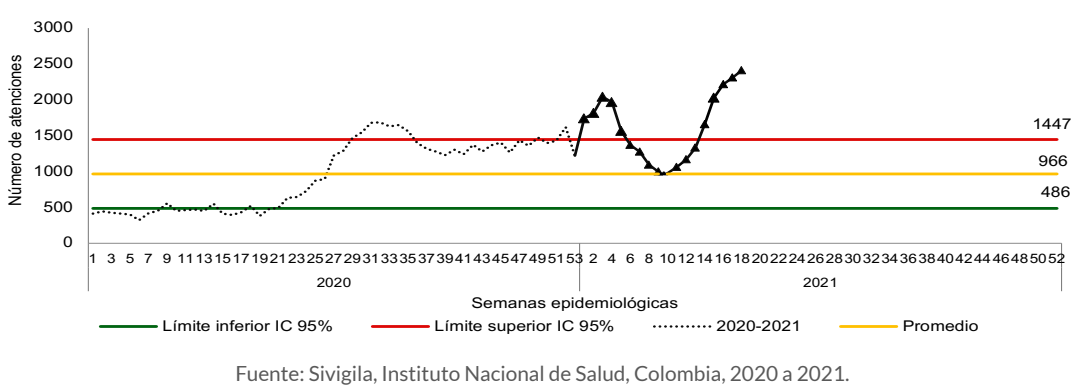

\section{METODOLOGÍA:}

Vigilancia de morbilidad por IRA: los canales endémicos para consultas externas y urgencias y hospitalizaciones en sala general se realizaron con la metodología de Bortman con los datos de la morbilidad por infección respiratoria aguda mediante el cálculo de la media geométrica de los años 2014 a 2020 y el intervalo de confianza.

Para las hospitalizaciones por IRAG en UCl e intermedio se construyó gráfico de control mediante el cálculo del promedio, la desviación estándar y el intervalo de confianza del año anterior.

\section{Dengue}

En la semana epidemiológica 18 de 2021 se notificaron 562 casos probables de dengue: 299 casos de esta semana y 263 casos de semanas anteriores. En el sistema hay 11996 casos, 6155 (51,3\%) sin signos de alarma, 5659 $(47,2 \%)$ con signos de alarma y $182(1,5 \%)$ de dengue grave.

En Colombia, los casos de dengue proceden de 32 departamentos, 5 distritos, 604 municipios. Las entidades territoriales de Cali, Valle del Cauca, Cartagena, Putumayo, Tolima, Huila, Norte de Santander, Meta, Antioquia, Cundinamarca, Cesar, Barranquilla, Santander, Atlántico y Bolívar aportan el 78,8 \% (9 451) de los casos a nivel nacional (tabla 8).
Tema central

Situación Nal.

Mortalidad

Trazadores

Brotes

COVID-19

Tablas 
Tabla 8. Casos notificados de dengue por entidad territorial de procedencia y clasificación en Colombia, semanas epidemiológicas 01 a 18 de 2021

\begin{tabular}{|c|c|c|c|c|c|c|}
\hline \multirow{2}{*}{ Entidad territorial } & \multicolumn{2}{|l|}{ Dengue } & \multicolumn{4}{|c|}{ Dengue grave Total } \\
\hline & $\bar{n}$ & $\%$ & $\mathrm{n}$ & $\%$ & $n$ & $\%$ \\
\hline Cali & 2402 & 20,3 & 29 & 15,9 & 2431 & 20,3 \\
\hline Valle del Cauca & 1078 & 9,1 & 10 & 5,5 & 1088 & 9,1 \\
\hline Cartagena & 672 & 5,7 & 16 & 8,8 & 688 & 5,7 \\
\hline Putumayo & 648 & 5,5 & 3 & 1,6 & 651 & 5,4 \\
\hline Tolima & 582 & 4,9 & 1 & 0,5 & 583 & 4,9 \\
\hline Huila & 542 & 4,6 & 14 & 7,7 & 556 & 4,6 \\
\hline Norte de Santander & 462 & 3,9 & 11 & 6,0 & 473 & 3,9 \\
\hline Meta & 458 & 3,9 & 2 & 1,1 & 460 & 3,8 \\
\hline Antioquia & 434 & 3,7 & 8 & 4,4 & 442 & 3,7 \\
\hline Cundinamarca & 411 & 3,5 & 1 & 0,5 & 412 & 3,4 \\
\hline Cesar & 377 & 3,2 & 19 & 10,4 & 396 & 3,3 \\
\hline Barranquilla & 355 & 3,0 & 9 & 4,9 & 364 & 3,0 \\
\hline Santander & 347 & 2,9 & 4 & 2,2 & 351 & 2,9 \\
\hline Atlántico & 276 & 2,3 & 6 & 3,3 & 282 & 2,4 \\
\hline Bolívar & 266 & 2,3 & 8 & 4,4 & 274 & 2,3 \\
\hline Magdalena & 224 & 1,9 & 9 & 4,9 & 233 & 1,9 \\
\hline Caquetá & 220 & 1,9 & 3 & 1,6 & 223 & 1,9 \\
\hline Sucre & 210 & 1,8 & 3 & 1,6 & 213 & 1,8 \\
\hline Córdoba & 205 & 1,7 & 2 & 1,1 & 207 & 1,7 \\
\hline Cauca & 198 & 1,7 & 0 & 0,0 & 198 & 1,7 \\
\hline Casanare & 196 & 1,7 & 0 & 0,0 & 196 & 1,6 \\
\hline Nariño & 156 & 1,3 & 6 & 3,3 & 162 & 1,4 \\
\hline Chocó & 158 & 1,3 & 1 & 0,5 & 159 & 1,3 \\
\hline Santa Marta & 148 & 1,3 & 6 & 3,3 & 154 & 1,3 \\
\hline Boyacá & 134 & 1,1 & 0 & 0,0 & 134 & 1,1 \\
\hline Amazonas & 133 & 1,1 & 0 & 0,0 & 133 & 1,1 \\
\hline Buenaventura & 96 & 0,8 & 1 & 0,5 & 97 & 0,8 \\
\hline Arauca & 86 & 0,7 & 0 & 0,0 & 86 & 0,7 \\
\hline Quindío & 73 & 0,6 & 2 & 1,1 & 75 & 0,6 \\
\hline Risaralda & 71 & 0,6 & 1 & 0,5 & 72 & 0,6 \\
\hline Caldas & 65 & 0,6 & 0 & 0,0 & 65 & 0,5 \\
\hline La Guajira & 36 & 0,3 & 4 & 2,2 & 40 & 0,3 \\
\hline Archipiélago de San Andrés & 30 & 0,3 & 0 & 0,0 & 30 & 0,3 \\
\hline Exterior & 27 & 0,2 & 2 & 1,1 & 29 & 0,2 \\
\hline Guaviare & 16 & 0,1 & 0 & 0,0 & 16 & 0,1 \\
\hline Vichada & 13 & 0,1 & 0 & 0,0 & 13 & 0,1 \\
\hline Guainía & 8 & 0,1 & 1 & 0,5 & 9 & 0,1 \\
\hline Vaupés & 1 & 0,0 & 0 & 0 & 1 & 0,0 \\
\hline Total & 11814 & 100 & 182 & 100 & 11996 & 100 \\
\hline
\end{tabular}

El 61,4 \% (7 366) de los casos de dengue se reportó en 39 municipios, con mayor frecuencia en: Cali, con 20,3 \% (2 431); Cartagena, con el 5,7 \% (688); Barranquilla, con 3,0 \% (364); Cúcuta, con el 2,4 \% (285); Orito, con el 1,8\% (221); Neiva, con el 1,4\% (166); Yumbo (161), Villavicencio (154), Santa Marta (154), Ibagué (153) y Aguachica (151) con el 1,3\% cada uno; y Villagarzón con el 1,1 \% (126).

Se hospitalizó el 76,0 \% (4 299) de los casos de dengue con signos de alarma y el $92,3 \%$ (168) de dengue grave; las entidades notificadoras que hospitalizaron menos del 60,0 \% de los casos de dengue con signos de alarma fueron: Boyacá, Valle del Cauca, Caldas, Buenaventura, Bolívar y Chocó; y las entidades notificadoras que no hospitalizaron el $100 \%$ de los casos de dengue grave fueron: Cali, Huila, Cartagena, Santa Marta, Magdalena, Santander, Valle del Cauca, La Guajira, Quindío y Cundinamarca.

A semana 18 se ha confirmado el 45,7\% (2 587) de los casos de dengue con signos de alarma, las entidades que han confirmado el 100,0 $\%$ de los casos notificados son Buenaventura y Vichada. Por otro lado, se ha confirmado el 56,6\% (103) de los casos de dengue grave y las entidades con el $100,0 \%$ de los casos confirmados son Buenaventura, Caquetá, Chocó, Córdoba, La Guajira, Risaralda y Sucre.

La incidencia nacional de dengue es de 38,8 casos por cada 100000 habitantes en riesgo. En las entidades de Amazonas, Putumayo, Cali, Cundinamarca, Cartagena, Caquetá y Archipiélago de San Andrés y Providencia se estiman tasas de incidencia superiores a 62,1 casos por 100000 habitantes (figura 11).

Fuente: Sivigila, Instituto Nacional de Salud, Colombia, 2021 
Figura 11. Incidencia de dengue por entidad territorial de procedencia en Colombia, semana epidemiológica 18 de 2021

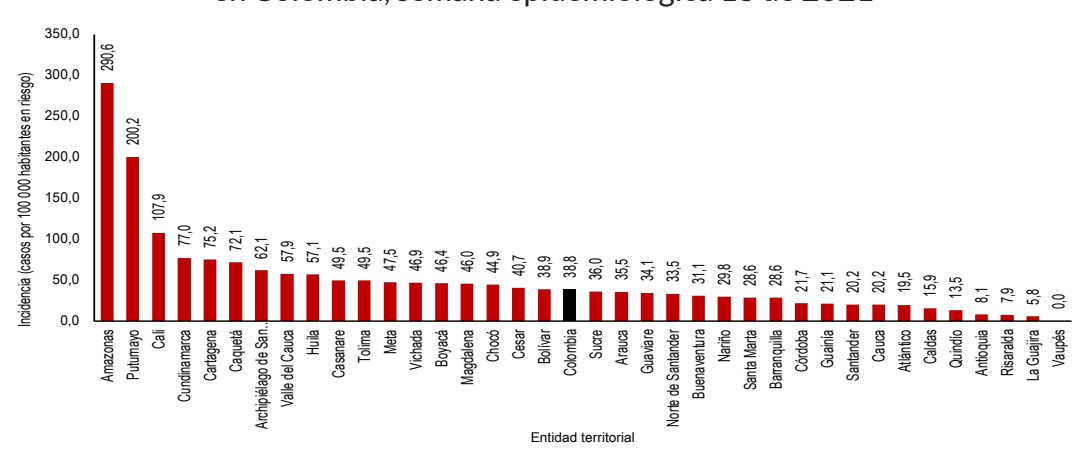

Fuente: Sivigila, Instituto Nacional de Salud, Colombia, 2021

A semana epidemiológica 18 de 2021, el evento a nivel nacional presentó un comportamiento dentro de lo esperado, comparado con su comportamiento histórico (figura 12).

Figura 12. Canal endémico nacional de dengue en Colombia, semana epidemiológica 18, 2021

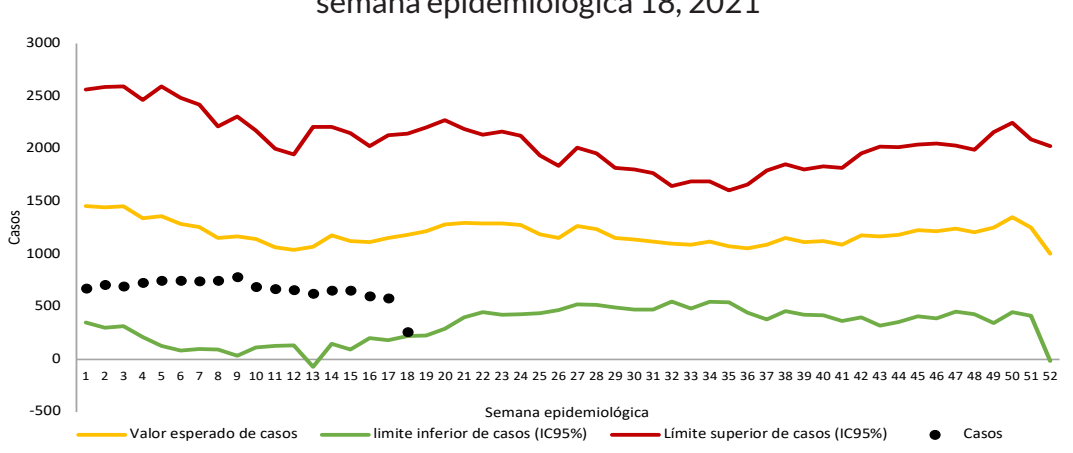

Fuente: Sivigila, Instituto Nacional de Salud, Colombia, 2021

De acuerdo con la situación epidemiológica según canal endémico, 21 entidades territoriales se encuentran dentro de lo esperado, 14 entidades se encuentran en situación de alerta y 2 entidades con presentación de casos por encima del valor esperado, comparado con el comportamiento histórico (tabla 9).
Tabla 9. Comparación de los casos notificados de dengue con su comportamiento histórico, por entidad territorial de procedencia en Colombia, semanas epidemiológicas 15 a 17 de 2021

\begin{tabular}{llll}
\hline $\begin{array}{l}\text { Comportamiento } \\
\text { epidemiológico }\end{array}$ & \multicolumn{3}{l}{ Entidad territorial } \\
\hline $\begin{array}{l}\text { Dentro del número } \\
\text { esperado de casos }\end{array}$ & Antioquia & Bolívar & Boyacá \\
& Casanare & Cali & Córdoba \\
& Caldas & Cundinamarca & Guainía \\
& Guaviare & La Guajira & Huila \\
& Meta & Nariño & Quindío \\
& Risaralda & Santander & Sucre \\
\cline { 2 - 4 } Tolima & Vaupés & Vichada \\
\hline Situación de alerta & Amazonas & Arauca & Atlántico \\
& Barranquilla & Caquetá & Cauca \\
& Cesar & Chocó & Magdalena \\
& Norte de Santander & Putumayo & Santa Marta \\
\cline { 2 - 4 } & Valle del Cauca & San Andrés, Providenciay Santa Catalina \\
\hline Por encima del número & Buenaventura & Cartagena & \\
esperado de casos & Fuente: Sivigita, Instituto Nacional de Salud, Colombia 2021 &
\end{tabular}

Durante el 2021 se han notificado 44 muertes probables por dengue, de las cuales 7 han sido confirmadas, procedentes de: Cartagena, con 5 casos; Barranquilla y Córdoba, con 1 caso cada uno. Se han descartado 8 casos y se encuentran en estudio 29 muertes procedentes de: Cesar y Magdalena, con 4 casos cada uno; Valle del Cauca y Huila, con 3 casos cada uno; Antioquia, Barranquilla, Quindío y Santa Marta, con 2 casos cada uno; Atlántico, Bolívar, Archipiélago de San Andrés y Providencia, Cali, Nariño, Vaupés y Exterior, con 1 caso cada uno.

\section{Metodología}

Se realizó un informe descriptivo de los casos notificados durante la semana epidemiológica 18 de 2021, teniendo en cuenta las variables de tiempo, persona y lugar contenidas en la ficha de datos básicos y complementarios del evento de dengue, dengue grave y mortalidad por dengue (Código INS 210, 220 y 580). Los indicadores se presentan en distribuciones de frecuencias en figuras y tablas.

El canal endémico nacional y el análisis de comportamiento epidemiológico por entidad territorial se realizó con la metodología de medias geométricas (Marcelo Bortman), estableciendo los siguientes límites de control: por debajo de lo esperado, número de casos menor al límite inferior IC $95 \%$; dentro de lo esperado, número de casos entre el límite inferior y la media geométrica IC95\%; en alerta, número de casos entre la media geométrica y el límite superior IC $95 \%$, e incremento por encima de lo esperado, número de casos por encima del límite superior IC $95 \%$.

En el análisis del comportamiento de dengue por canal endémico no se tiene en cuenta la semana epidemiológica actual dado que, el periodo de incubación del virus de dengue es de 3 a 14 días, por lo tanto, los casos de esta semana se reflejarán plenamente en la siguiente semana. 


\section{Malaria}

\section{Análisis epidemiológico nacional}

Según el análisis del último periodo epidemiológico, el país se encuentra en situación de seguridad para malaria, como lo muestra el canal endémico (Figura 13).

Figura 13. Canal endémico de malaria, Colombia, semana epidemiológica 18, 2021

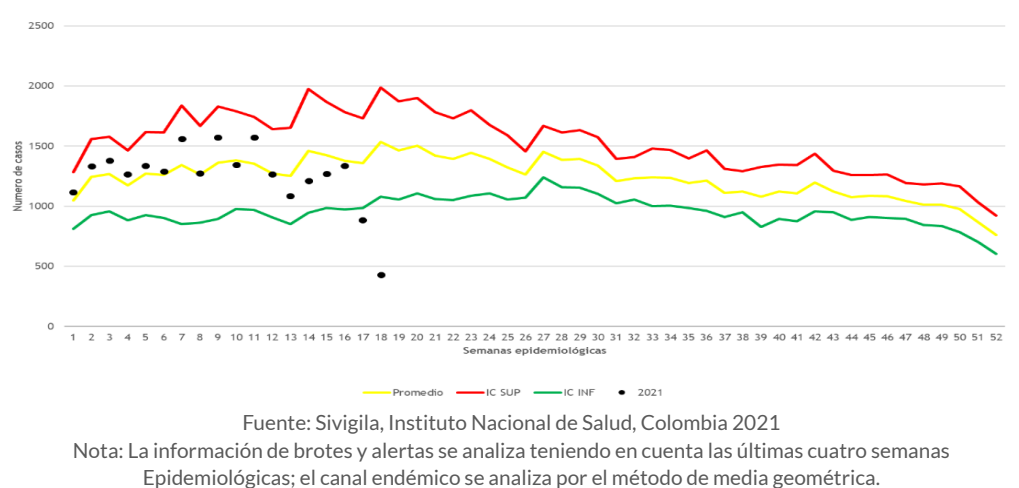

En la semana epidemiológica 18 se notificaron 1107 casos de malaria, teniendo un acumulado de 22764 casos, de los cuales 22 312 son de malaria no complicada y 452 de malaria complicada. Predomina la infección por Plasmodium falciparum (P. falciparum) con 50,9\% (11 597), seguido de Plasmodium vivax (P. vivax) con 48,3 \% (10 989) e infección mixta con 0,8 \% (178).

\section{Malaria no complicada}

Por procedencia, Chocó (30,7\%), Nariño (24,3\%), Córdoba (12,7\%), Antioquia (9,7\%) y Guainía (4,5\%) aportan el $81,9 \%$ de los casos de malaria no complicada (Tabla 10).
Tabla 10. . Casos notificados de malaria no complicada por entidad territorial de procedencia. Colombia, semanas epidemiológicas 01 a 17 de 2021

\begin{tabular}{|c|c|c|c|c|c|c|}
\hline Entidad territorial & Infección mixta & P. falciparum & P. malarie & P. vivax & $\mathrm{n}$ & $\%$ \\
\hline Chocó & 62 & 3637 & 0 & 3143 & 6842 & 30,70 \\
\hline Nariño & 23 & 4845 & 0 & 551 & 5419 & 24,30 \\
\hline Córdoba & 11 & 585 & 0 & 2243 & 2839 & 12,70 \\
\hline Antioquia & 15 & 663 & 0 & 1482 & 2160 & 9,70 \\
\hline Guainía & 4 & 65 & 0 & 933 & 1002 & 4,50 \\
\hline Cauca & 1 & 907 & 0 & 16 & 924 & 4,10 \\
\hline Vichada & 16 & 140 & 0 & 397 & 553 & 2,50 \\
\hline Buenaventura & 3 & 386 & 0 & 65 & 454 & 2,00 \\
\hline Norte de Santander & 1 & 1 & 0 & 452 & 454 & 2,00 \\
\hline Guaviare & 2 & 62 & 0 & 329 & 393 & 1,80 \\
\hline Risaralda & 2 & 11 & 0 & 302 & 315 & 1,40 \\
\hline Bolívar & 15 & 36 & 0 & 238 & 289 & 1,30 \\
\hline Exterior & 4 & 25 & 0 & 207 & 236 & 1,06 \\
\hline Meta & 0 & 30 & 0 & 163 & 193 & 0,90 \\
\hline Amazonas & 0 & 1 & 0 & 74 & 75 & 0,30 \\
\hline Casanare & 0 & 0 & 0 & 55 & 55 & 0,20 \\
\hline Vaupés & 0 & 1 & 0 & 18 & 19 & 0,10 \\
\hline Cali & 0 & 11 & 0 & 5 & 16 & 0,10 \\
\hline Valle del Cauca & 0 & 5 & 0 & 5 & 10 & 0,00 \\
\hline Putumayo & 0 & 1 & 0 & 8 & 9 & 0,00 \\
\hline Desconocido & 0 & 3 & 0 & 5 & 8 & 0,04 \\
\hline Santander & 1 & 1 & 0 & 6 & 8 & 0,00 \\
\hline Sucre & 0 & 1 & 0 & 7 & 8 & 0,00 \\
\hline La Guajira & 0 & 0 & 0 & 6 & 6 & 0,00 \\
\hline Cesar & 2 & 1 & 0 & 3 & 6 & 0,00 \\
\hline Arauca & 0 & 0 & 0 & 5 & 5 & 0,00 \\
\hline Cartagena & 0 & 0 & 0 & 4 & 4 & 0,00 \\
\hline Huila & 0 & 0 & 0 & 3 & 3 & 0,00 \\
\hline Caquetá & 0 & 1 & 0 & 1 & 2 & 0,00 \\
\hline Magdalena & 0 & 1 & 0 & 1 & 2 & 0,00 \\
\hline Barranquilla & 1 & 0 & 0 & 0 & 1 & 0,00 \\
\hline Atlántico & 0 & 0 & 0 & 1 & 1 & 0,00 \\
\hline Total & 163 & 11420 & 0 & 10729 & 22312 & 100 \\
\hline
\end{tabular}

\section{Malaria Complicada}

Se notificaron 452 casos de malaria complicada, que proceden de 23 entidades territoriales y 6 casos provienen del exterior. Nariño, Antioquia, Chocó, Córdoba, Cauca, Meta y Vichada notifican el 80,2 \% de los casos.

De los casos de malaria complicada el 60,4\% (273) corresponde a hombres. El 29,6\% (134) se presenta en personas de 15 a 24 años y el 19,0\% (86) ocurre en indígenas. Por lugar de procedencia, el 40,5\% (183) proviene de rural disperso. Por régimen de afiliación, el 66,8 \% (302) pertenece al régimen subsidiado. 
De los casos de malaria complicada causados por P. vivax o P. falciparum, el 77,7 \% (351) presentó complicaciones hematológicas, el 17,0 \% (77) complicaciones hepáticas, el 5,3\% (24) complicaciones renales, el 2,9 \% (13) complicaciones pulmonares y el 2,4\% (11) malaria cerebral (Figura 14).

Figura 14. Tipo de complicaciones de malaria, Colombia, semanas epidemiológicas 01 a 18 de 2021

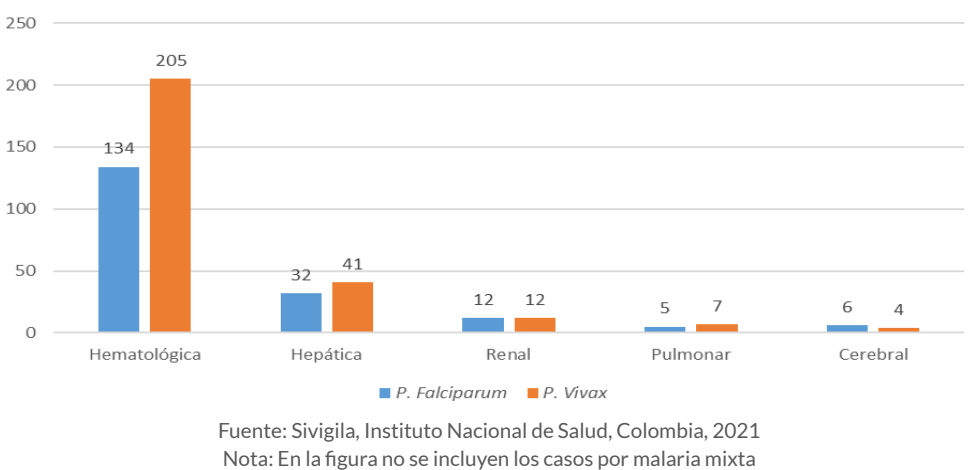

\section{Comportamientos inusuales}

A semana epidemiológica 18, un departamento está por encima del número esperado de casos y cuatro departamentos están en situación de alerta para malaria (Tabla 11).

Tabla 11. Distribución de entidades territoriales según el comportamiento epidemiológico de malaria, Colombia, semana epidemiológica 18 de 2021

\begin{tabular}{|c|c|c|c|}
\hline Comportamiento epidemiológico & Departamentos & & \\
\hline Menor al comportamiento histórico & Amazonas & Antioquia & \\
\hline En el comportamiento histórico & La Guajira & Arauca & Atlántico \\
\hline & Caquetá & Quindío & Caldas \\
\hline & Huila & Sucre & Magdalena \\
\hline & $\begin{array}{l}\text { Cesar } \\
\text { Risaralda } \\
\text { Valle del Cauca } \\
\text { Guainía }\end{array}$ & $\begin{array}{l}\text { Chocó } \\
\text { Vichada } \\
\text { Putumayo } \\
\text { Norte de Santander } \\
\text { Guaviare }\end{array}$ & $\begin{array}{l}\text { Santander } \\
\text { Bolívar } \\
\text { Casanare } \\
\text { Vaupés }\end{array}$ \\
\hline Situación de alerta & Risaralda & $\begin{array}{l}\text { Nariño } \\
\text { Cauca }\end{array}$ & Córdoba \\
\hline Mayor al comportamiento histórico & Meta & & \\
\hline
\end{tabular}

Nota: La información de brotes y alertas se analiza teniendo en cuenta las últimas cuatro semanas epidemiológicas
A semana epidemiológica 18, 15 municipios se encuentran en situación de brote; los que más reportan casos son: Bajo Baudó (Chocó), El Charco, Olaya Herrera y Roberto Payán (Nariño) (Tabla 12).

\begin{tabular}{lcccc}
\multicolumn{5}{c}{$\begin{array}{c}\text { Tabla 12. Municipios en situación de brote por malaria } \\
\text { a semana epidemiológica 18 en Colombia, 2021 }\end{array}$} \\
\cline { 2 - 5 } \multicolumn{1}{c}{ Departamento } & Municipio & Acumulado & Esperado & Observado \\
\cline { 2 - 5 } Chocó & Bajo Baudó & 1424 & 108 & 356 \\
Nariño & El Charco & 857 & 114 & 225 \\
Nariño & Olaya Herrera & 729 & 99 & 266 \\
Nariño & Roberto Payán & 710 & 155 & 183 \\
Nariño & Mosquera & 497 & 38 & 115 \\
Cauca & Guapi & 444 & 83 & 138 \\
Chocó & Medio San Juan & 443 & 79 & 206 \\
Risaralda & Pueblo Rico & 319 & 41 & 69 \\
Nariño & La Tola & 183 & 16 & 37 \\
Córdoba & Montería & 147 & 1 & 52 \\
Chocó & Litoral Del Bajo San Juan & 107 & 22 & 44 \\
Meta & Mapiripán & 91 & 5 & 11 \\
Meta & Puerto Rico & 31 & 4 & 7 \\
Nariño & Policarpa & 27 & 3 & 3 \\
Meta & Puerto López & 7 & 1 & 5 \\
& Fuente: Sivigila, Instituto Nacional de Salud, Colombia 2021 & \\
Nota: La información de brotesyalertas se analiza teniendo en cuentalas últimas cuatro semanas epidemiológicas
\end{tabular}

\section{Comportamientos inusuales (municipios IREM)}

La Iniciativa Regional para la Eliminación de la Malaria (IREM) se implementa en doce municipios del Pacífico colombiano, con el objetivo de reducir y eliminar la transmisión de la malaria. Busca fortalecer la red de diagnóstico para propender por un diagnóstico y tratamiento oportuno de casos de malaria, plantea la vigilancia en salud pública como intervención en salud pública y busca cortar cadenas de transmisión.

A semana epidemiológica 18, de los 12 municipios en los que se implementa la IREM, según el análisis de comportamientos inusuales, ninguno se encuentra en incremento. Se encuentran en decremento: Bagadó, Juradó, Quibdó, Riosucio, Unguía y Buenaventura, ninguno se encuentra en situación de brote.

\section{Tema central \\ Situación Nal. \\ Mortalidad \\ Trazadores \\ Brotes \\ COVID-19}


Tabla 13. Comportamientos inusuales, municipios Iniciativa Regional para la Eliminación de la Malaria, semana epidemiológica 18, Colombia, 2021

\begin{tabular}{lllllll}
\hline Departamento & Municipios & Acumulado & Esperado & Observado & $\begin{array}{c}\text { Comportamiento } \\
\text { inusual }\end{array}$ & $\begin{array}{c}\text { Situación } \\
\text { epidemiológica }\end{array}$ \\
& Acandí & 14 & 8 & 4 & Ninguno & Seguridad \\
& Atrato & 156 & 69 & 52 & Ninguno & Seguridad \\
& Bagadó & 311 & 127 & 97 & Disminución & Seguridad \\
& Juradó & 9 & 10 & 2 & Disminución & Seguridad \\
\multirow{5}{*}{ Chocó } & Lloró & 165 & 146 & 44 & Ninguno & Éxito \\
& Medio Atrato & 217 & 114 & 55 & Ninguno & Seguridad \\
& Quibdó & 1397 & 761 & 199 & Disminución & Éxito \\
& Rioquito & 101 & 66 & 20 & Ninguno & Seguridad \\
& Riosucio & 60 & 12 & 2 & Disminución & Seguridad \\
& Unguía & 6 & 9 & 1 & Disminución & Seguridad \\
\hline Buenaventura a & Buenaventura & 462 & 146 & 112 & Disminución & Éxito \\
\hline Nariño & Tumaco & 689 & 164 & 123 & Ninguno & Seguridad \\
\hline
\end{tabular}

Fuente: Sivigila, Instituto Nacional de Salud, Colombia 2021

Nota: La información de brotes y alertas se analiza teniendo en cuenta las últimas cuatro semanas epidemiológicas

\section{Metodología}

Se realizó un análisis descriptivo con corte a semana epidemiológica 18 de 2021, con información que incluye: descripción de los casos en tiempo, lugar y persona, análisis de tendencia, comportamientos inusuales, descripción yanálisis de indicadores parala vigilancia.

\section{BROTES Y SITUACIONES DE EMERGENCIA EN SALUD PÚBLICA}

\section{ALERTAS NACIONALES}

\section{Brote de Enfermedad transmitida por alimentos, Solano - Caquetá.}

Se notificó brote de enfermedad transmitida por alimentos el 12 de mayo, cuyo inicio fue el 2 de mayo de 2021 con 14 enfermos, 59 expuestos (tasa de ataque de $23,7 \%$ ), quienes presentaron un cuadro clínico caracterizado por nauseas, vómito, diarrea, cefalea, deshidratación y dolor abdominal. No se reportan fallecidos ni hospitalizaciones. Los alimentos probablemente implicados fueron pastas con pollo, de las que no se pudo obtener muestras. Acciones realizadas: se verificaron las condiciones locativas encontrando como factores de riesgo, la limitación al transporte de los alimentos a las instalaciones; las condiciones de calor y humedad del sitio geográfico e interferencias en la cadena de frío relacionadas con el transporte. Se dictaron recomendaciones. Situación en cierre.

\section{Eventos de control internacional}

En Colombia en la semana epidemiológica 19 no se reportaron casos o alertas de enfermedad por el virus Ébola, peste bubónica/ neumónica ni carbunco; sin embargo, se continúa el monitoreo de alertas y el fortalecimiento de acciones de vigilancia, notificación y respuesta a nivel nacional y subnacional.

\section{ALERTAS INTERNACIONALES}

Actualización epidemiológica semanal sobre COVID-19. Fecha de Publicación 11 de mayo 2021. Organización Mundial de la Salud (OMS)

El número de casos de COVID-19 a nivel mundial ha presentado una leve disminución esta semana con más de 5,5 millones de nuevos casos y más de 90000 muertes. La incidencia de casos y muertes, sin embargo, permanece en los niveles más altos desde el inicio de la pandemia. Los casos nuevos disminuyeron en la región de Europa y el Mediterráneo oriental, mientras que la región del Sudeste
Tema centra

Situación $\mathrm{Nal}$

Mortalidad

Trazadores

Brotes

COVID-19

Tablas 
Asiático continua con un aumento por novena semana consecutiva con un 6 \% comparado con la semana anterior. La incidencia de fallecidos incrementó en el Sudeste Asiático y el Pacífico occidental, India continúa aportando el $95 \%$ de los casos y el $93 \%$ de las muertes de la región del Sudeste Asiático, así como el 50\% de los casos y el $30 \%$ de las muertes a nivel mundial, observándose tendencias preocupantes en los países vecinos.

El mayor número de nuevos casos se reportaron en la India (2 738 957 nuevos casos; incremento del 5\%), Brasil (423 438 casos nuevos; similar a la semana anterior), Estados Unidos (334 784 nuevos casos; disminución del 3\%), Turquía (166 733 casos nuevos; disminución del 35\%) y Argentina (140 771 nuevos casos, con disminución del 8\%).

\section{Variantes SARS-CoV-2}

Las actividades de vigilancia para la detección de variantes de SARSCoV-2 se han fortalecido a nivel local y nacional, incluidas las estrategias de secuenciación genómica, el número de países/territorios/ áreas que reportan variantes de interés y variantes de preocupación (VOI y VOC siglas en ingles respectivamente) continúa incrementando. Desde la actualización del 4 de mayo, VOC 202012/01 se ha reportado en siete nuevos países, la variante 501Y.V2 en cinco países y la variante P1 en cuatro nuevos países. Hasta el 11 de mayo, un total de 149 países ha reportado VOC 202012/01, 102 países la variante $501 Y . V 2$ y 60 la variante P1. La información presentada debe ser interpretada de acuerdo con las limitaciones de la vigilancia incluidas la capacidad de secuenciación y priorización de muestras entre los diferentes países.

\section{Nueva designación de VOC al linaje B.1.617}

En consulta con el grupo de trabajo de la OMS sobre la evolución del virus SARS-CoV-2, el cual ha determinado que los virus con linaje B.1.617 ha sido caracterizados como VOC B.1.617, contiene tres sublinajes con algunas diferencias, pero potencialmente relevantes mutaciones en la proteína pico, así como en la prevalencia de la detección a nivel mundial. Al 11 de mayo, más de 4500 secuencias han sido reportados al GISAID y se asignó al B.1.617 en 44 países de las 6 regiones de la OMS, y se han recibido informes de detección de cinco países adicionales. Se designó B.1.617 como VOC basado en la temprana evidencia de impactos fenotípicos en comparación con otras variantes de los virus circundantes, especialmente porque los sublinajes de B.1.617 parecen tener tasas más altas de transmisión, incluidos los aumentos rápidos observados en la prevalencia en varios países (evidencia moderada B.1.617.1 y B.1.617.2) y la evidencia preliminar sugiere una posible reducción en la eficacia de Bamlanivimab, un anticuerpo monoclonal utilizado para el tratamiento de COVID-19, y una susceptibilidad potencialmente levemente reducida a los anticuerpos neutralizantes (evidencia limitada disponible para B.1.617.1).

Se requieren con urgencias más estudios solidos sobre el impacto fenotípico de estas variantes incluidos los impactos sobre las características epidemiológicas (transmisibilidad, gravedad, riesgos de reinfección entre otros) y el impacto sobre las contramedidas.

\section{Panorama regional de la OMS}

Región de África. La región reporto alrededor de 40000 casos nuevos y más de 1000 nuevas muertes la última semana, con una disminución del $5 \%$ y el incremento del 3\% respectivamente, comparado con la semana anterior. Esto sigue una tendencia a la baja a largo plazo en la incidencia de casos y muertes, sin embargo, esta tendencia puede revertirse pronto con el aumento de casos y muertes en algunos países. El mayor número de casos nuevos se notificó en Sudáfrica (11 975 nuevos casos, un aumento del 11\%), Etiopía (4 155 casos nuevos, una disminución del 42\%) y Camerún (4 126 casos, disminución del 10\%). El mayor número de nuevas muertes se registró en Sudáfrica (318 muertes, una disminución del 32\%), Etiopia (162 nuevas muertes, una disminución del 9\%) y Kenia (139 nuevas muertes, disminución del 10\%)

Región de las Américas. La región ha reportado más de 1,2 millones de casos nuevos y más de 33000 nuevas muertes con una disminución del $4 \%$ y $8 \%$ respectivamente comparado con la semana anterior. Esta es la tercera semana consecutiva de disminución de la incidencia de casos en la región, sin embargo, los casos y las muertes continúan aumentando en algunos países. El mayor número de caso nuevos se reportó Brasil (423 438 casos nuevos; similar a la semana anterior), Estados Unidos de América (334 784 casos nuevos; un 
3\% de disminución) y Argentina (140 711 casos nuevos; una disminución del 8\%). El mayor número de nuevas muertes se registró en Brasil (15 333 nuevas muertes; una disminución del 3\%), los Estados Unidos de América (4 940 nuevas muertes, un incremento del 4\%) y Colombia (3 147 nuevas muertes; una disminución del 4\%).

Región del Mediterráneo Oriental. La región notifico alrededor de 280000 nuevos casos y más de 5600 nuevas muertes, ambas tasas disminuyeron un $13 \%$ en comparación con la semana anterior. Esta primera semana se ha reportado una marcada disminución después de 11 semanas de aumento de casos. El mayor número de casos nuevos se notificó en República Islámica de Irán (124 513 casos nuevos; un decremento del 10\%), Iraq (38 192 casos nuevos; una disminución del 10\%) y Pakistán (28 721 casos nuevos; una disminución del 19\%). El mayor número de nuevas muertes se registró en la República Islámica de Irán (2 434 nuevas muertes; una disminución del 18\%), Pakistán (840 nuevas muertes; una disminución de 12\%) y Túnez (542 nuevas muertes; una disminución del 6\%).

Región Europea. La región ha reportado cerca de 897000 nuevos casos y un poco menos de 19000 nuevas muertes con una disminución del $25 \%$ y $18 \%$ respectivamente comparado con la semana anterior. Se ha presentado una disminución en el número de nuevos casos y muertes el último mes. El mayor número de casos nuevos se notificó en Turquía (166 733 casos nuevos; una disminución del $35 \%$ ), Francia (122 487 casos nuevos; una disminución del 26\%) y Alemania (103 507 casos nuevos; una disminución del 20\%). El mayor número de nuevas muertes se registró en la Federación Rusa (2 464 nuevas muertes; una disminución del 6\%), Turquía (2 242 nuevas muertes; una disminución del 10\%) y Polonia (1 944 nuevas muertes; una disminución del 4\%).

Región de Asia Sudoriental. En la última semana, la región del Sudeste Asiático notificó cerca de 2.8 millones nuevos casos y 29000 nuevas muertes, un aumento del $6 \%$ y del $15 \%$, respectivamente, en comparación con la semana anterior. Esta es la novena semana consecutiva en que la incidencia de casos y muertes ha ido en aumento en la región. El mayor número de casos fue reportado por India (2 738957 casos nuevos; un aumento del 5\%), Nepal (56 997 casos nuevos; un aumento del 79\%) e Indonesia (36 882 casos nuevos; un incremento de $2 \%)$. El mayor número de nuevas muertes se registró en India (26 820 nuevas muertes; un aumento del 15\%), Indonesia (1 190 nuevas muertes; un aumento del 3\%) y Bangladesh. (368 nuevas muertes; una disminución del 34\%).

Región del Pacífico Occidental. La semana anterior se notificaron más 127000 nuevos casos y poco más de 1700 nuevas muertes, una disminución del $4 \%$ y un aumento del $34 \%$, respectivamente, en comparación con la semana anterior. El mayor número de casos nuevos se notificó en Filipinas (48 197 casos nuevos; un decremento del 16\%), Japón (35 805 casos nuevos; un aumento del 2\%), y Malasia ( 25350 casos nuevos, un incremento del 19\%) El mayor número de nuevas muertes se registró en Filipinas (915 nuevas muertes; un incremento 35\%), Japón (527 nuevas muertes; un aumento del $38 \%$ ) y Malasia (136 nuevas muertes; un incremento del $43 \%$ ).

Fuente: Organización Mundial de la Salud (OMS). Actualización epidemiológica semanal de COVID-19. Fecha de Publicación 11 de mayo de 2021. Fecha de consulta 13 de mayo de 2021. Disponible en: https://www.who.int/publications/m/item/weekly-epidemiological-update-on-covid-19---11-may-2021

\section{Boletín de brotes y emergencias. Guinea, Brote Enfermedad Virus del Ébola (EVE) 03 -09 de mayo de 2021, semana 19. Organización Mun- dial de la Salud (OMS)}

No se han confirmado nuevos casos de enfermedad por virus del ébola (EVE) reportados por la prefectura de N'Zerekore, sitio del actual brote de EVE hasta el 9 de mayo de 2021. No hay subprefecturas activas dentro de la prefectura de N'Zerekore, y no se han notificado nuevos casos en los últimos 21 días. Sin embargo, hay 4 nuevos casos sospechosos de los cuales se tomó muestra a uno y tres se han negado a tomarse muestra.

Al 9 de mayo de 2021, un total de 23 casos han sido reportados, incluidos 16 casos confirmados y 7 casos probables, de los cuales nueve se han recuperado con una tasa de recuperación del 39,1\%. De los 23 casos, 12 han fallecido (letalidad 52,2\%). El número de trabajadores de salud infectados son cinco. El caso confirmado perdido continúa desaparecido, la cuenta regresiva para el fin del brote es hasta el 8 de mayo 2021, incluido este caso. La mayoría de los ca- 
sos confirmados y probables son mujeres (13/23: $60,9 \%$ ) y el grupo de edad más afectado es alrededor de los 40 años.

Un total de 1114 contactos han sido seguidos y el 56\% de ellos han sido vacunados. Al 9 de mayo de 2021 un total de 35 nuevas alertas han sido reportadas en N'Zerekore, incluidas tres muertes. De estas alertas 10 (29\%) fueron investigadas en las primeras 24 horas y seis fueron validadas. Un total de 29 alertas fueron validadas y seis de los fallecidos se les tomo muestra. Se recibieron seis nuevas alertas en las prefecturas vecinas, todas muertes en la comunidad, todas fueron investigas y ninguna fue validada.

La cuenta regresiva de 42 días para el fin el brote comenzó el 8 de mayo de 2021, con la fecha prevista para la declaración del fin del brote el 19 de junio de 2021. Las acciones de respuesta en salud publica incluyen reuniones de seguimiento y coordinación en un centro de operaciones de emergencia recientemente equipado con el apoyo de la OMS. Un acumulado de 9569 personas han sido vacunadas, incluidos 622 contactos de alto riesgo, 8413 contactos de contactos, incluidos 2694 trabajadores de primera línea. Un caso sospechoso ha sido hospitalizado en Centros de tratamiento para enfermedades epidémicas en N'zerekore y un caso sospechoso fue hospitalizado en Goueke, un total de cuatro pacientes, todos sospechosos han sido manejados en centros de tratamiento.

Las actividades de prevención y control de infecciones incluidas una sesión de orientación sobre los principios de bioseguridad y gestión de los casos sospechosos sobre EVE para 40 trabajadores sanitarios de cuatro lugares de aislamiento en N'zerekore. Así como una sesión informativa sobre la norma de EVE y precauciones suplementarias para seis trabajadores de la salud del Centro de Salud de Koropara.

Fuente: Boletín de brotes y emergencias. Guinea, Brote Enfermedad Virus del Ébola (EVE) 03 - 09 de mayo de 2021, semana 19. Organización Mundial de la Salud (OMS). Fecha de publicación 9 de mayo de 2021. Fecha de consulta 13 de mayo de 2021. Disponible en: https://www.afro.who.int/health-topics/disease-outbreaks/ outbreaks-and-other-emergencies-updates
Brote por síndrome neurológico de causa desconocida - Canadá. Informe de amenazas de enfermedades trasmisibles 9-15 de mayo de 2021. Centro europeo para la prevención y el control de enfermedades (ECDC)

Un brote por síndrome neurológico de causa desconocida ha sido reportado en Nueva Brunswick, Canadá. Al 6 de mayo se han reportado 48 casos, incluidas seis muertes. En algunos casos se necesita más información para determinar su la causa de muertes fue un resultado de este síndrome. La mayoría de los casos tiene fecha de inicio de síntomas entre 2018-2020 excepto un caso que experimento síntomas en 2013 la proporción de sexo es de 1:1 y la edad oscila entre 18 y 85 años.

La mayoría de los casos viven en las regiones sureste y noreste de Nueva Brunswick, sin embargo, hasta ahora no hay evidencia que sugiera que los residentes de estas regiones corran mayor riesgo. Algunos síntomas incluyen problemas de memoria, espasmos musculares, problemas de equilibrio, dificultad para caminar o caídas, visión borrosa o alucinaciones visuales inexplicables y pérdida significativa de peso, cambios de comportamiento. La definición de caso se finalizó el 5 de marzo de 2021 y se envió a los médicos de Nueva Brunswick para fomentar la búsqueda de casos.

El sistema de vigilancia de enfermedades de Creutzfeldt-Jakob (CJDSS) de la Agencia de Salud Pública de Canadá ha investigado activamente la posibilidad de enfermedad priónica humana, pero hasta la fecha todas las pruebas han sido negativas para formas conocidas de esta enfermedad.

Fuente: Brote de síndrome neurológico de causa desconocida - Canadá. Informe de amenazas de enfermedades trasmisibles 9-15 de mayo de 2021. Centro europeo para la prevención y el control de enfermedades (ECDC). Fecha de publicación 12 de mayo de 2021. Fecha de consulta 13 de mayo de 2021. Disponible en: https:// www.ecdc.europa.eu/en/publications-data/communicable-disease-threats-report-9-15-may-2021-week-19 


\section{SITUACIÓN COVID-19}

Hasta el 13 de mayo de 2021 se han confirmado por laboratorio 3 067879 casos de COVID-19 en Colombia, en 38 entidades territoriales del orden departamental y distrital, afectando 1116 municipios. La incidencia acumulada es de 6 090,39 casos por cada 100 000 habitantes. Las entidades territoriales que superan esta incidencia son: Barranquilla, Bogotá, Santa Marta, Amazonas, Cali, Antioquia, Quindío, Cartagena, San Andrés, Caldas, Atlántico y Risaralda (figura 1). A la fecha el 3,4 \% (103 304) de los casos se encuentra activo, siendo procedente principalmente de Bogotá con el 47,2\% (48 747), Antioquia con el 16,6\% (17 206), Santander con el 4,4\% (4 598), Cundinamarca con el 4,1\% (4 245), Valle del Cauca con el 4,0 \% (4 147) y Barranquilla D.E. con el 3,5 \% (3 694). A nivel municipal (con excepción de Bogotá, Barranquilla, Cartagena y Santa Marta), el 17,3\% (18 214) de los casos activos se encuentra principalmente en: Medellín (9 375), Cali (2 796), Bucaramanga (2 070), Bello (1 506), Itagüí (1 405) y Valledupar (1 062).

La incidencia nacional de casos activos es de 205,15 casos por 100 000 habitantes. Los municipios y distritos con mayor incidencia de casos activos son: Bogotá $(629,4)$, Armenia-Antioquia (512,4), Málaga-Santander (484,4), Itagüí-Antioquia $(484,4)$, Caldas-Antioquia $(451,9)$, Apartadó-Antioquia $(403,9)$ y Rionegro-Antioquia $(401,4)$. De los casos activos el 52,7 \% (54 451) se reportó en mujeres y según el grupo de edad el $71,1 \%$ (73 440) se presenta en personas entre los 20 y los 59 años. De acuerdo con la ubicación, el 86,6 \% (89 515) de los casos activos se encuentra en casa, el 10,6 \% (10 949) hospitalizado en sala general y el 2,7 \% (2 840) hospitalizado en $\mathrm{UCl}$ (mapa 1).
Figura 13. Incidencia acumulada y de casos activos de COVID-19 por entidad territorial de procedencia, Colombia, 13 de mayo de 2021

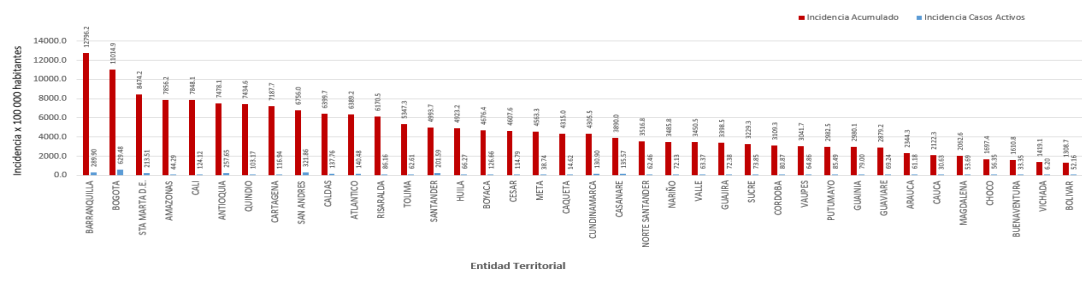

Fuente: INS- Base Reporte COVID-19, 13 de mayo 2021

Entre el 11 de abril y el 8 de mayo de 2021 (SE 15 - 18, 2021), se reportaron 304838 casos casos de COVID-19, para una incidencia de 639,18 casos por 100000 habitantes. Las entidades con incidencias superiores a la nacional fueron: Atlántico $(836,47)$, BarranquiIla (2 186,97), Bogotá, D.C. (1 427,10), Cartagena (682,63), Caldas $(728,36)$, Santa Marta $(1015,64)$, Archipiélago de San Andrés, Providencia y Santa Catalina (1 080,79). Para este periodo, comprendido entre las semanas epidemiológicas (SE) 15 a 18 de 2021, se presentó un aumento significativo de la incidencia respecto al periodo anterior en las entidades territoriales de Bogotá, D.C., Bolívar, Cartagena, Boyacá, Caquetá, Cauca, Cundinamarca, Chocó, Nariño, Norte de Santander, Santander, Tolima, Buenaventura, Arauca, Casanare, Putumayo, Archipiélago de San Andrés, Providencia y Santa Catalina y Guaviare. Las siguientes entidades territoriales registraron un descenso significativo en la incidencia de COVID-19 en comparación con el periodo anterior: Antioquia (0,8), Atlántico $(0,5)$, Barranquilla $(0,5)$, Caldas (0,8), Cesar (0,9), Córdoba (0,7), La Guajira (0,5), Magdalena $(0,5)$, Santa Marta $(0,5)$, Quindío $(0,6)$, Risaralda $(0,7)$, Sucre $(0,8)$, Valle del Cauca $(0,7)$, Cali $(0,5)$, Amazonas $(0,4)$, Guainía $(0,6)$, Vaupés $(0,9)$ y Vichada $(0,6)$ (mapa 1$)$.
Tema central

Situación $\mathrm{Nal}$

Mortalidad

Trazadores

Brotes

COVID-19

Tablas 
Mapa 1. Incidencia de casos COVID-19 por entidad territorial, Colombia, semana epidemiológica 15 a 18, 2021

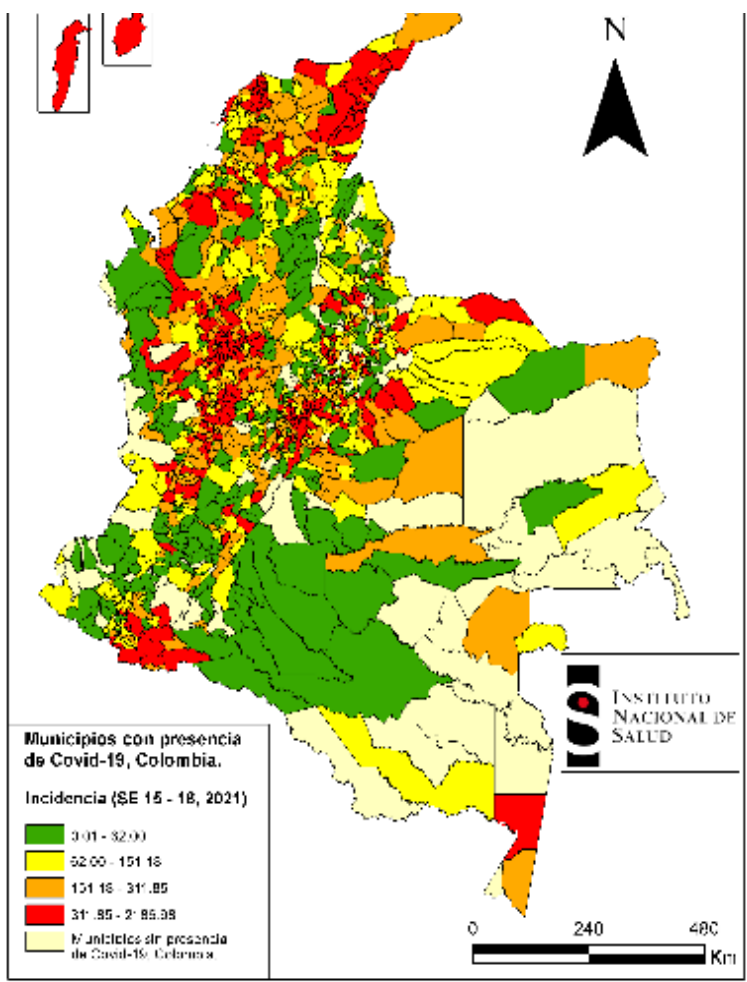

Fuente: INS- Sala de Análisis del Riesgo COVID-19, 2021

Hasta la fecha se han reportado 79760 fallecidos por COVID-19, entre el 11 de abril y el 8 de mayo de 2021 (SE 15 - 18, 2021) se notificaron 11635 fallecidos, lo que corresponde al 14,6\% del total de casos fatales. Durante el periodo analizado (SE 15-18, 2021) fallecieron 906 personas entre los 15 y 44 años y 8462 personas mayores de 60 años, que corresponden al 7,8\% y $72,4 \%$ de las muertes del periodo (11 635); durante este tiempo se notificaron 9 casos de muerte por esta causa en menores de 5 años.

Las entidades con mayor notificación de fallecidos durante este periodo epidemiológico (SE 15-18, 2021) fueron: Antioquia con 244,0 \%
(2 794), Bogotá D.C. con 14,9 \% (1 744), Barranquilla con 13,0 \% (1 515), Atlántico con 7,1 \% (779), Cali con 4,3\% (511) y Cundinamarca con 4,0 \% (472). Entre las semanas epidemiológicas (SE) 15 a 18, 2021, los municipios y distritos con la mayor tasa de mortalidad fueron: Barranquilla (119,24), Hobo - Huila (95,02), Puerto Colombia - Atlántico (93,37), Baranoa - Atlántico (85,19), Antioquia - Caldas $(78,1)$ Santo Tomás - Atlántico $(72,06)$ y Soledad - Atlántico $(67,92)$ (mapa 2).

Mapa 2. Mortalidad por COVID-19 por entidad territorial, Colombia, semana epidemiológica 13 a 16, 2021

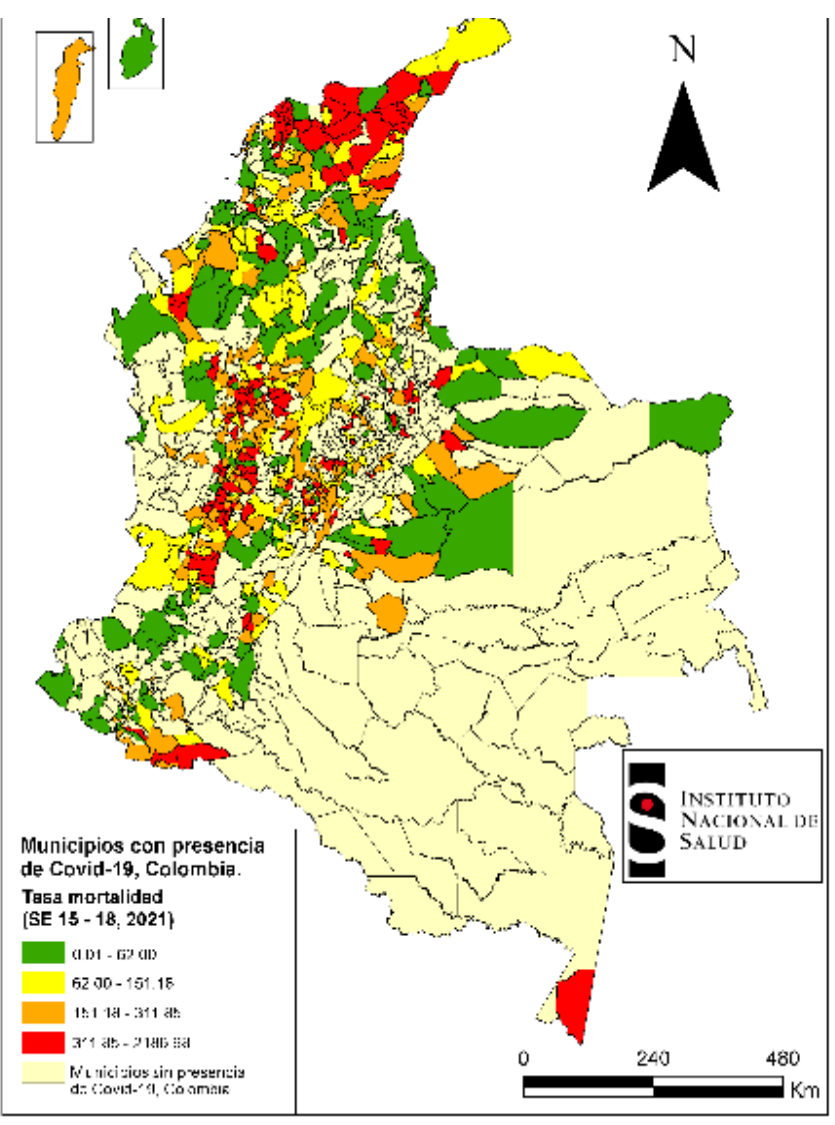

Fuente: INS- Sala de Análisis del Riesgo COVID19, 2021
Tema central

Situación $\mathrm{Na}$

Mortalidad

Trazadores

Brotes

COVID-19

Tablas 
A nivel nacional, al comparar la tasa de mortalidad y letalidad de la SE 15 a 18, 2021 respecto al periodo anterior (SE 11-14, 2021), se observó que de los 498 municipios que reportaron muertes por COVID-19 durante el periodo analizado el 78,5 \% (391) presentó incremento significativo en la tasa de mortalidad y letalidad; de estos municipios los que presentaron mayor variación (aumento) en la tasa de mortalidad fueron Norcasia - Caldas $(\triangle 350,47)$, Bojacá - Cundinamarca $(\Delta 334,73)$, San Carlos de Guaroa - Meta $(\Delta 296,15)$, Suan - Atlántico ( $\Delta 296,08)$, La Jagua del Pilar - La Guajira $(\Delta 283,29)$, y los municipios que presentaron mayor variación (aumento $\Delta 100,0$ ) en la tasa de letalidad fueron Abriaquí - Antioquia, Anorí - Antioquia), Puerto Triunfo - Antioquia, Santo Domingo - Antioquia Panqueba - Boyacá , San Miguel de Sema - Boyacá , Zetaquira - Boyacá , Belén de los Andaquíes - Caquetá , Almaguer - Cauca, Florencia - Caquetá, Guapi - Cauca, Villa Rica - Cauca , Chimá - Córdoba , Chaguaní - Cundinamarca, La Peña - Cundinamarca, Une - Cundinamarca , Baraya - Huila , Saladoblanco - Huila, El Dorado - Meta , Guamal - Meta , Imués - Nariño, Policarpa - Nariño, Ricaurte Nariño, Sapuyes - Nariño, Pamplonita - Norte de Santander, Puerto Santander - Norte de Santander, Albania - Santander, Bolívar - Santander, Coromoro - Santander, El Guacamayo - Santander , Ocamonte - Santander , Ambalema-Tolima, Carmen de Apicalá Tolima, Roncesvalles - Tolima, Orocué - Casanare.

Durante las SE 15 a 18 de 2021, 91 municipios presentaron un incremento significativo de los indicadores para COVID-19, $8 \mathrm{mu}-$ nicipios presentaron incremento de la incidencia, tasa de mortalidad, letalidad, positividad y un descenso o patrón estable de tasa de muestreo; 18 municipios registraron aumento de la incidencia, tasa de mortalidad, letalidad, positividad y tasa de muestreo con reporte de fallecidos por COVID-19 en el periodo anterior (SE 11 - 14, 2021) y 65 municipios reportaron aumento de la incidencia, tasa de mortalidad, letalidad, positividad y tasa de muestreo sin fallecidos por COVID-19 en el periodo anterior (SE 10 - 13, 2021).
Tabla 14. Clasificación de municipios según prioridades para COVID-19, Colombia, semana epidemiológica 15 a 18, 2021

Clasificación Municipios

Prioridad 1:

municipios con aumento

incidencia, tasa de mortalidad,

incidencia, tasade mortalidad,

letalidad, positividad y descenso 0

Prioridad 2.1: municipios con aumento incidencia, tasa de mortalidad, letalidad, positividad $y$ tasa de muestreo con fallecidos por COVID-19 en el periodo anterior

\section{Prioridad 22. municipios con} aumento incidencia, tasa de mortalidad, letalidad, positividad y tasa de muestreo sin fallecidos por COVID-19 en el periodo anterior

\section{Antioquia (Caramanta), Bolívar (El Peñón, San Jacinto del Cauca), Cauca (Patía), Santander (Barichara, Rionegro), Valle del Cauca (Guacarí, Vijes).}

Antioquia (Caucasia, Sopetrán, Támesis), Atlántico (Manati), Bolivar (El Carmen Magangué), Cundinamarca (Funza), Magdalena (Guamal, San Sebastián de Buenavista), Risaralda (Belén de Umbría), Santander (Floridablanca, Girón), Sucre (San Marcos) Valle del Cauca (Buenaventura Cartago La Victoria) Putum, Sucre (Orito, Puerto Asis)

Antioquia (Amagá, Andes, El Bagre, Nechí, Pueblorrico, Sabanalarga, San Andrés de Cuerquia, Segovia), Atlántico (Repelón), Boyacá (Güicán de la Sierra, Puerto Boyacá, Soatá, Susacón), Caldas (Palestina, Riosucio, Salamina, Viterbo), Cesar (Pelaya, San Martín), Cundinamarca (Agua de Dios, Caparrapí, Guaduas, La Peña, Pacho, Quipile, Tocaima, Ubaque, Une, Villapinzón), Chocó (El Cartón de San Pablo, Nuquí, Tadó), Huila (Algeciras, Isnos, Suaza, Villavieja), La Guajira (La Jagua del Pilar), Meta (El Dorado, Puerto Rico), Nariño (Imués, Linares, Puerres, Sapuyes),

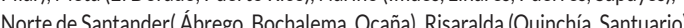
Sartede Santander (Bolivar, El Carmen de Chucuri, EI Peñon, Guepsa, Lebrija, San Andrés) Sucre (Sucre), Tolima (Icononzo, Prado, Purificación, Venadillo), Valle del Cauca (Ginebra), Arauca (Fortul), Casanare (Nunchía, Pore), Putumayo (San Miguel, Valle del Guamuez).

Fuente: INS- Sala de Análisis del Riesgo COVID19, 2021

Durante las últimas cuatro semanas epidemiológicas (SE 15 - 18), 2021 se han notificado por fecha de inicio 141conglomerados COVID-19, de los cuales 141 (100\%) se encuentran activos con 913 casos confirmados y 3646 muestras obtenidas para un porcentaje de positividad del $25,04 \%$.

De los conglomerados activos, el 36,9\% (52) se presentó en empresa con 253 casos confirmados; 20,6 \% (29) en Fuerzas Militares con 331 casos confirmados; $13,5 \%$ (19) en ámbito comunitario con 93 casos; $9,2 \%$ (13) en centros de protección con 80 casos; $6,4 \%$ (9) en instituciones educativas con 40 casos; $4,3 \%$ (6) en población carcelaria con 38 casos; $2,8 \%$ (4) en migrantes con 12 casos; $2,8 \%$ (4) en instituciones de salud con 24 casos; $2,1 \%$ (3) en indígenas con 32 casos y $1,4 \%$ (2) en policía con diez casos confirmados. 


\section{TABLAS DE MANDO NACIONAL}

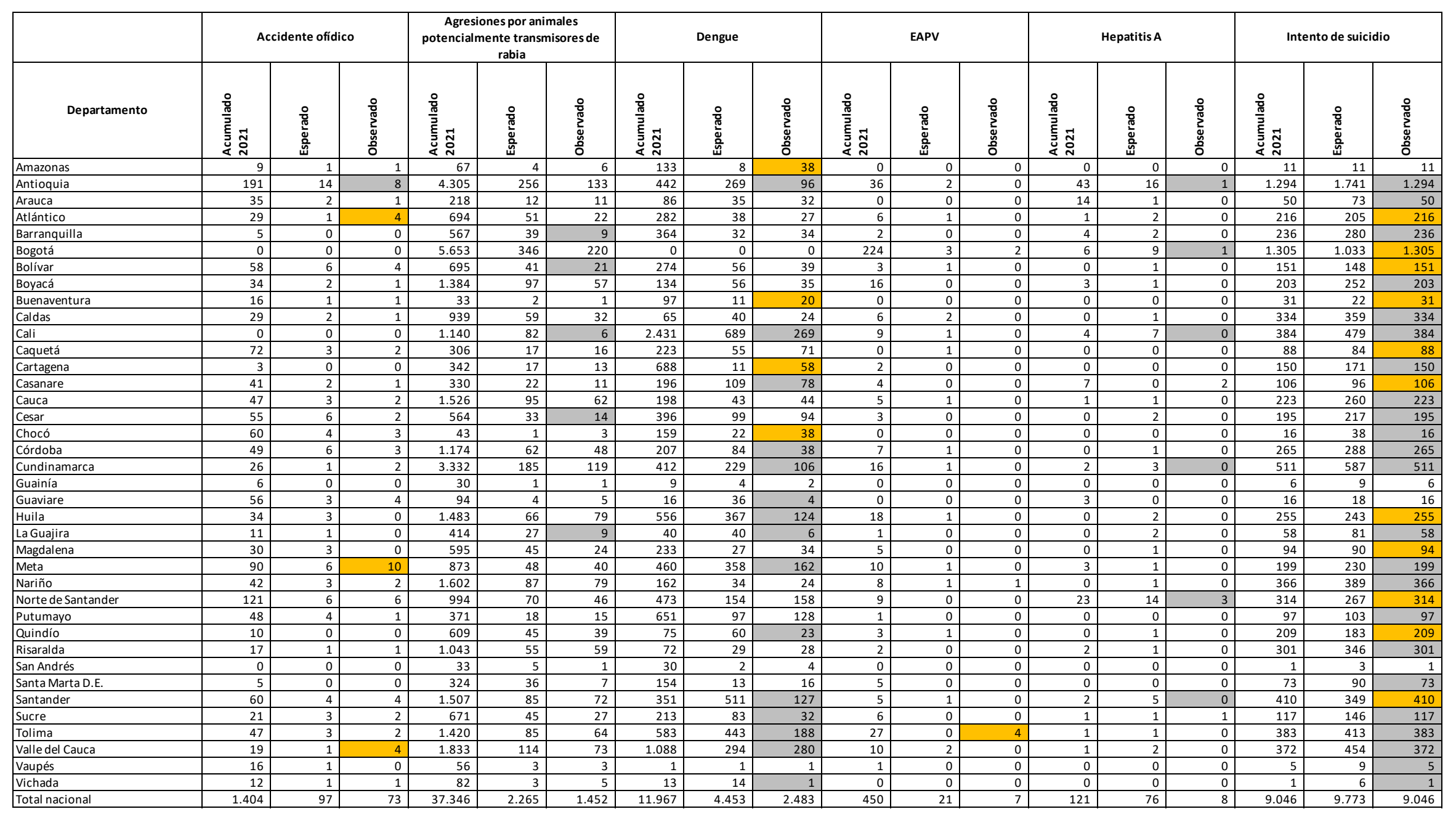

Tema central

Situación Nal.

Mortalidad

Trazadores

Brotes

COVID-19

Tablas 


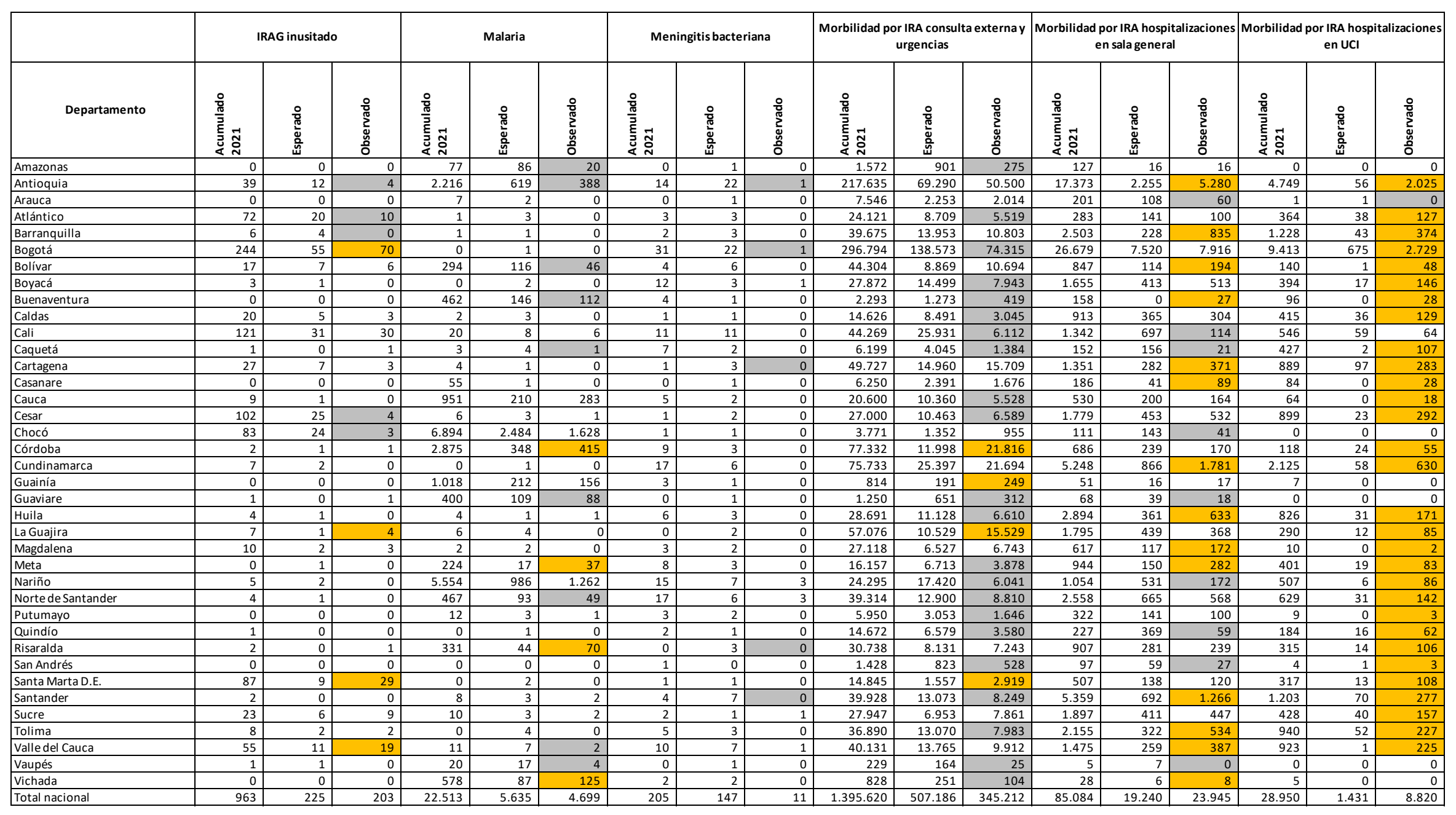

Tema central

Situación Nal.

Mortalidad

Trazadores

Brotes

COVID-19

Tablas 


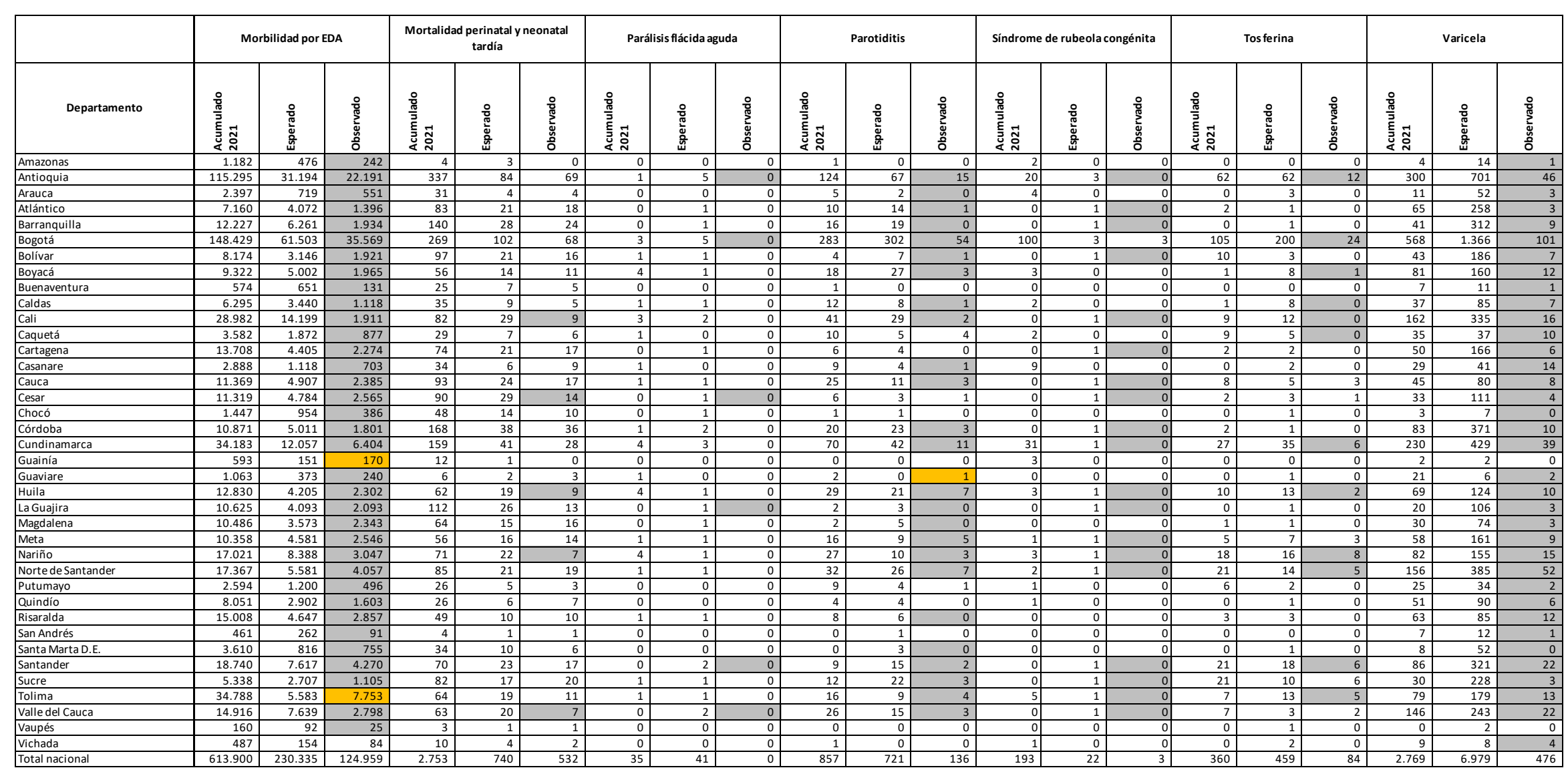

Tema central

Situación Nal.

Mortalidad

Trazadores

Brotes

COVID-19

Tablas 


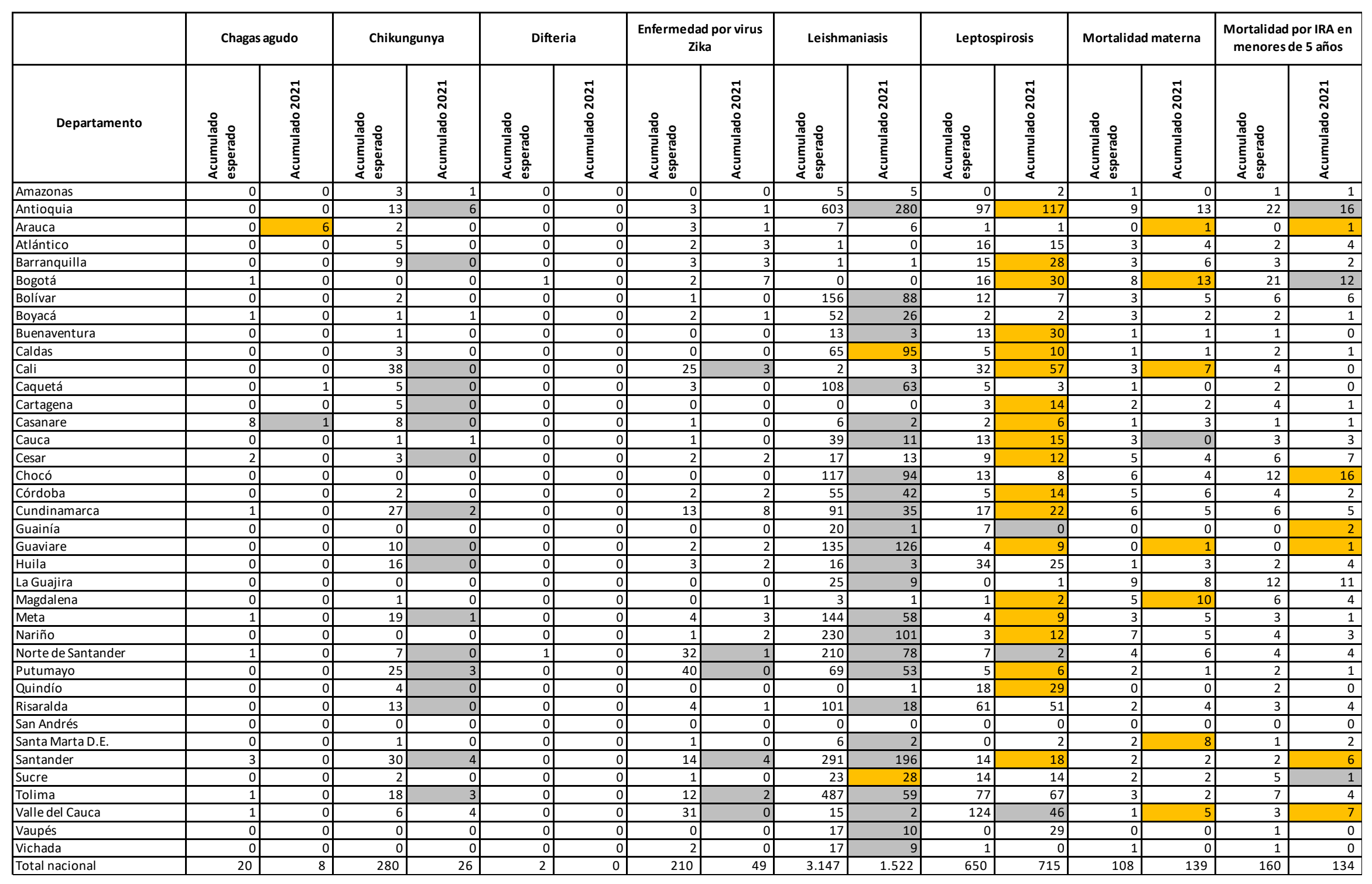




\section{Comportamiento de la notificación por departamento a semana 18}

\begin{tabular}{|c|c|c|c|c|c|c|c|c|c|c|c|c|c|c|c|c|}
\hline \multirow[b]{2}{*}{ Departamento } & \multicolumn{2}{|c|}{$\begin{array}{c}\text { Mortalidad por y } \\
\text { asociada a DNT en } \\
\text { menores de } 5 \text { años }\end{array}$} & \multicolumn{2}{|c|}{ Sarampión/Rubeola } & \multicolumn{2}{|c|}{ Sífilis congénita } & \multicolumn{2}{|c|}{ Sifilis gestacional } & \multicolumn{2}{|c|}{ Tétanos accidental } & \multicolumn{2}{|c|}{ Tuberculosis } & \multicolumn{2}{|c|}{$\begin{array}{c}\text { Tuberculosis } \\
\text { farmacorresistente }\end{array}$} & \multicolumn{2}{|c|}{$\begin{array}{l}\text { Violencia de género e } \\
\text { intrafamiliar }\end{array}$} \\
\hline & $\begin{array}{l}\frac{0}{0} \\
\frac{\pi}{3} \\
\frac{\pi}{5} \\
\frac{\pi}{2} \\
\overline{3} \\
\frac{3}{4}\end{array}$ & 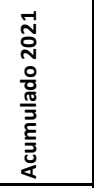 & 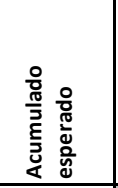 & 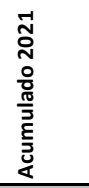 & 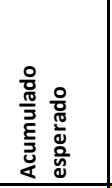 & 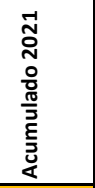 & 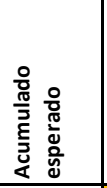 & 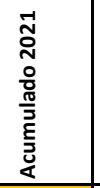 & 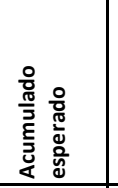 & 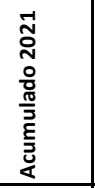 & 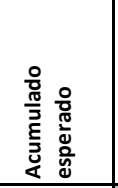 & 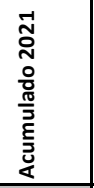 & 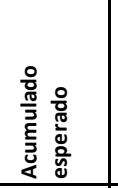 & 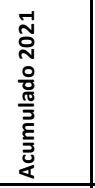 & 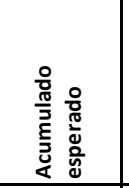 & 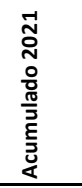 \\
\hline \begin{tabular}{|l|} 
Amazonas \\
\end{tabular} & 0 & 0 & 1 & 0 & 1 & 4 & 5 & 9 & 0 & 0 & 21 & 11 & 0 & 0 & 111 & 67 \\
\hline Antioquia & 4 & 3 & 48 & 16 & 39 & 57 & 219 & 357 & 1 & 1 & 924 & 825 & 33 & 26 & 5.699 & 4.095 \\
\hline \begin{tabular}{|l|} 
Arauca \\
\end{tabular} & 0 & 0 & 2 & 1 & 5 & 29 & 25 & 89 & 0 & 0 & 39 & 51 & 1 & 2 & 240 & 263 \\
\hline Atlántico & 2 & 1 & 10 & 1 & 11 & 16 & 63 & 133 & 1 & 0 & 135 & 103 & 10 & 1 & 630 & 638 \\
\hline \begin{tabular}{|l|} 
Barranquilla \\
\end{tabular} & 1 & 0 & \begin{tabular}{l|l}
9 \\
\end{tabular} & 5 & 16 & 33 & 51 & 165 & 0 & 1 & 214 & 190 & 0 & 2 & 418 & 519 \\
\hline Bogotá & 0 & 0 & 55 & 197 & 49 & 49 & 291 & 312 & 0 & 0 & 385 & 358 & 9 & 14 & 3.447 & 4.940 \\
\hline \begin{tabular}{|l|} 
Bolívar \\
\end{tabular} & 3 & 1 & 8 & 0 & 10 & 15 & 49 & 123 & 1 & 1 & 43 & 50 & 0 & 0 & 708 & 781 \\
\hline Boyacá & 0 & 0 & \begin{tabular}{l|l}
9 \\
\end{tabular} & 1 & 4 & 11 & 21 & 30 & 0 & 1 & 44 & 33 & 1 & 0 & 1.010 & 931 \\
\hline Buenaventura & 0 & 0 & 2 & 0 & 3 & 12 & 34 & 96 & 0 & 0 & 75 & 49 & 11 & 1 & 73 & 96 \\
\hline \begin{tabular}{|l|} 
Caldas \\
\end{tabular} & 0 & 0 & 7 & 4 & 5 & 10 & 34 & 53 & 0 & 0 & 111 & 79 & \begin{tabular}{l|l}
1 \\
\end{tabular} & 1 & 820 & 828 \\
\hline \begin{tabular}{|l|} 
Cali \\
\end{tabular} & 0 & 0 & 16 & 0 & 13 & 10 & 127 & 108 & 0 & 0 & 419 & 271 & 15 & 8 & 2.209 & 1.808 \\
\hline \begin{tabular}{|l|} 
Caquetá \\
\end{tabular} & 1 & 2 & 3 & 0 & 3 & 9 & 22 & 30 & 0 & 0 & 63 & 65 & 2 & 0 & 312 & 265 \\
\hline \begin{tabular}{|l|} 
Cartagena \\
\end{tabular} & 0 & 0 & 7 & 1 & 14 & 35 & 46 & 109 & 1 & 1 & 108 & 98 & 1 & 1 & 333 & 259 \\
\hline Casanare & 0 & 0 & 3 & 0 & 2 & 14 & 24 & 55 & 0 & 0 & 42 & 57 & 1 & 0 & 528 & 283 \\
\hline Cauca & 1 & 1 & 10 & 0 & 9 & 4 & 35 & 74 & 0 & 0 & 92 & 83 & 1 & 0 & 888 & 902 \\
\hline Cesar & 6 & 5 & 9 & 3 & 7 & 18 & 81 & 179 & 1 & 1 & 110 & 104 & 1 & 1 & 794 & 842 \\
\hline Chocó & 4 & 4 & 4 & 0 & 13 & 8 & 36 & 82 & 0 & 0 & 86 & 50 & 1 & 0 & 88 & 79 \\
\hline \begin{tabular}{|l|} 
Córdoba \\
\end{tabular} & 2 & 5 & 13 & 1 & 11 & 20 & 77 & 168 & 3 & 2 & 93 & 96 & 1 & 1 & 852 & 1.016 \\
\hline Cundinamarca & 1 & 0 & 24 & 2 & 17 & 38 & 71 & 146 & 0 & 0 & 136 & 106 & 2 & 4 & 2.409 & 2.685 \\
\hline \begin{tabular}{|l|} 
Guainía \\
\end{tabular} & 1 & 1 & 0 & 0 & 1 & 4 & 1 & 8 & 0 & 0 & 4 & 7 & 0 & 0 & 23 & 20 \\
\hline \begin{tabular}{|l|} 
Guaviare \\
\end{tabular} & 0 & 0 & 1 & 0 & 0 & 5 & 5 & 12 & 0 & 0 & 12 & 6 & 0 & 0 & 74 & 76 \\
\hline \begin{tabular}{|l|} 
Huila \\
\end{tabular} & 2 & 1 & 8 & 9 & 4 & 7 & 41 & 69 & 1 & 0 & 127 & 151 & 1 & 2 & 1.603 & 1.959 \\
\hline \begin{tabular}{|l|} 
La Guajira \\
\end{tabular} & 15 & 15 & 7 & 0 & 16 & 38 & 47 & 124 & 1 & 1 & 111 & 85 & 1 & 0 & 396 & 362 \\
\hline \begin{tabular}{|l|} 
Magdalena \\
\end{tabular} & 3 & 6 & 6 & 0 & 6 & 9 & 59 & 89 & 2 2 & 2 & 49 & 41 & 1 & 0 & 440 & 543 \\
\hline Meta & 2 & 3 & 7 & 0 & 11 & 31 & 47 & 112 & 0 & 0 & 168 & 173 & 4 & 4 & 602 & 606 \\
\hline \begin{tabular}{|l|} 
Nariño \\
\end{tabular} & 3 & 2 & 11 & 17 & 6 & 5 & 63 & 85 & 1 & 0 & 60 & 50 & 3 & 0 & 1.233 & 1.072 \\
\hline Norte de Santander & 2 & 1 & 11 & 11 & 8 & 44 & 44 & 225 & 0 & 0 & 173 & 148 & 3 & 3 & 1.016 & 1.088 \\
\hline \begin{tabular}{|l|} 
Putumayo \\
\end{tabular} & 1 & 1 & 3 & 1 & 2 & 2 & 16 & 41 & 0 & 0 & 34 & 22 & 0 & 0 & 282 & 326 \\
\hline Quindío & 0 & 0 & 4 & 0 & 3 & 4 & 32 & 40 & 0 & 0 & 77 & 68 & 1 & 0 & 593 & 699 \\
\hline Risaralda & 1 & 0 & 7 & 0 & 5 & 12 & 51 & 63 & 0 & 0 & 188 & 167 & 7 & 4 & 868 & 1.027 \\
\hline San Andrés & 0 & 0 & 0 & 0 & 0 & 0 & 1 & 1 & 0 & 0 & 5 & 0 & 0 & 0 & 24 & 20 \\
\hline Santa Marta D.E. & 0 & 2 & 4 & 1 & 3 & 9 & 21 & 40 & 0 & 0 & 56 & 60 & 0 & 2 & 192 & 187 \\
\hline \begin{tabular}{|l|} 
Santander \\
\end{tabular} & 0 & 0 & 16 & 6 & 15 & 21 & 83 & 164 & 0 & 1 & 227 & 218 & 5 & 6 & 2.511 & 1.834 \\
\hline Sucre & 0 & 3 & 7 & 5 & 8 & 11 & 58 & 94 & 1 & 2 & 29 & 29 & 0 & 1 & 529 & 591 \\
\hline \begin{tabular}{|l|} 
Tolima \\
\end{tabular} & 0 & 0 & 9 & 2 & 11 & 13 & 41 & 55 & 0 & 0 & 169 & 153 & 1 & 1 & 599 & 797 \\
\hline Valle del Cauca & 4 & 4 & 14 & 3 & 8 & 9 & 72 & 99 & 1 & 0 & 206 & 151 & 5 & 3 & 1.769 & 1.839 \\
\hline \begin{tabular}{|l|} 
Vaupés \\
\end{tabular} & 0 & 0 & 0 & 0 & 0 & 0 & 0 & 2 & 0 & 0 & 4 & 3 & 0 & 0 & 44 & 72 \\
\hline \begin{tabular}{|l|} 
Vichada \\
\end{tabular} & 3 & 0 & 1 & 0 & 0 & 1 & 3 & 10 & 0 & 0 & 7 & 13 & 0 & 1 & 56 & 36 \\
\hline Total nacional & 62 & 61 & 357 & 287 & 339 & 617 & 1.996 & \begin{tabular}{|l|}
3.651 \\
\end{tabular} & \begin{tabular}{l|l}
15 & \\
\end{tabular} & 14 & 4.835 & \begin{tabular}{|l|}
4.224 \\
\end{tabular} & \begin{tabular}{l|l}
117 & \\
\end{tabular} & 89 & \begin{tabular}{|l|l|}
34.423 \\
\end{tabular} & 34.451 \\
\hline
\end{tabular}

Tema central

Situación Nal.

Mortalidad

Trazadores

Brotes

COVID-19

Tablas

2 Instrtuto

La información es notificada semanalmente por las entidades territoriales (ET) al Instituto Nacional de Salud (INS) a través del

Sistema de vigilancia en salud pública (Sivigila). El número de casos, puede variar después de que se realizan unidades de 
INSTITUTO

NACIONAL DE

SALUD
Semana epidemiológica 18

2 al 8 de mayo de 2021

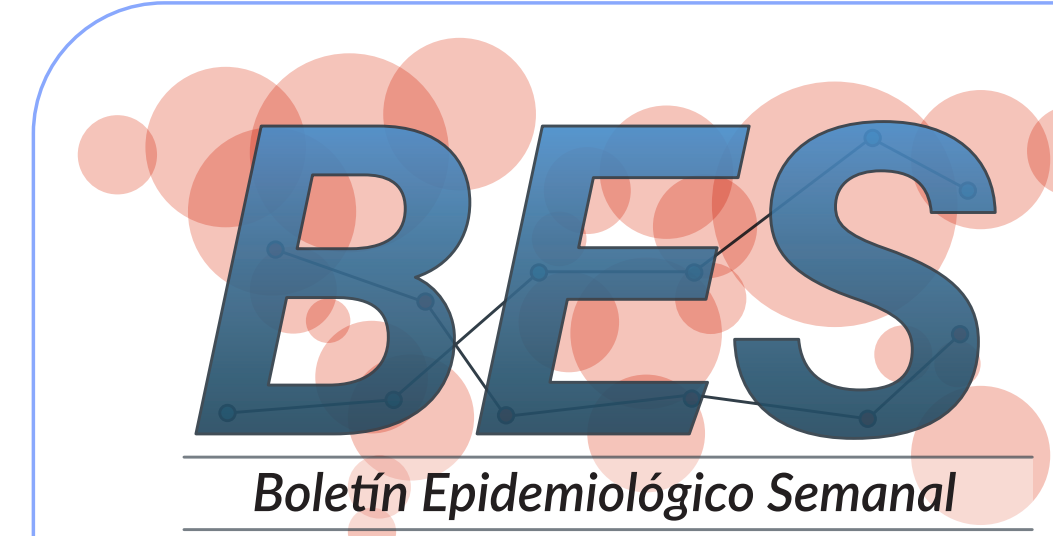

Expertos Temáticos

Tema Central

Profesional en entrenamiento FETP

Alejandra Romero Profesional GGRRICR

Dra. Martha Lucia Ospina Martínez

\section{Situación Nacional}

Grupo Sivigila sivigila@ins.gov.co

Mortalidad

Dr. Franklyn Edwin Prieto Alvarado

Dra. Diana Walteros

Una publicación del:

Instituto Nacional de Salud Dirección de Vigilancia y Análisis del Riesgo en Salud Pública Publicación en línea: ISSN 2357-6189 https://doi.org/10.33610/23576189.2021.18

Asistencia técnica de:

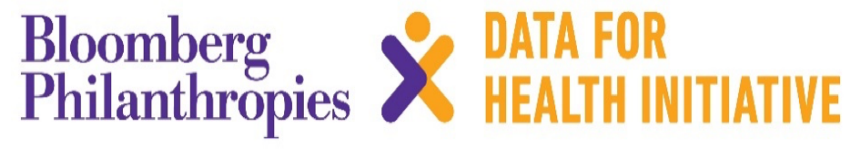

Giana María Henriquez Mendoza ghenriquez@ins.gov.co

Maria Eugenia Pinilla Saraza mpinillas@ins.gov.co

Diana Alexa Forero Motta dforero@ins.gov.co

Eventos Trazadores

Andrea Jineth Rodriguez Reyes arodriguezr@ins.gov.co

Carolina Ferro Mendez cferro@ins.gov.co

Brotes

Gestor sistema de alerta temprana eri@ins gov.co

Jorge Díazjidiaz@ins.gov.co

Tablas de mando

Yudy Silva Lizarazo ysilva@ins.gov.co

Diana Rivera drivera@ins.gov.co

Editor

Dr. Hernán Quijada Bonilla hquijada@ins.gov.co

Diseño y diagramación

Alexander Casas acasasc@ins.gov.co
La salud

es de todos 